\title{
A NEW MULTI-STAGE TURBINE STATOR DESIGN FOR IMPROVED PERFORMANCE RETENTION
}

\author{
Heather K. Jameson and John P. Longley \\ Whittle Laboratory \\ University of Cambridge \\ 1 J.J. Thomson Avenue \\ Cambridge CB3 ODY, UK
}

\section{ABSTRACT}

An experimental and computational investigation has been undertaken into the effects of deterioration of the first stage rotor shroud knife-edge seal clearance in a two-stage turbine which has engine representative cavity geometries. Four values of deterioration were investigated which cover the new-condition to old-condition knife-edge seal clearance.

Measurements within the first stage rotor shroud cavity show that whilst the leakage mass flow rate increases with deterioration, the angle at which the leakage flow approaches the downstream stator is essentially fixed and independent of the flow coefficient. This is in agreement with a simple over-tip leakage model. Because of the engine representative cavity geometry, the over-shroud leakage flow undergoes little mixing when it re-enters the mainstream and approaches the downstream stator at more than $60^{\circ}$ negative incidence.

Detailed measurements at the exit of the second stage stator identified two large positive vortices which were not consistent with the horseshoe vortex model for secondary flow. A computational investigation revealed that one vortex originates from the rolling-up within the stator passage of the streamwise vorticity sheet associated with the first stage rotor over-shroud leakage. This roll-up vortex cannot be eliminated. The second vortex is generated within the stator passage by the separation of the over-shroud leakage flow at the leading-edge due to the large negative incidence. It was hypothesised that this separation vortex might be eliminated by locally redesigning the stator.

A new stator was designed, manufactured and tested. As predicted, the roll-up vortex was still present but the separation vortex was eliminated. For all the values of deterioration tested the entropy loss coefficient of the new stator and the unchanged second stage rotor were reduced. It is estimated that the new stator would improve the lifetime average efficiency by $0.5 \%$ compared to the original.

\section{INTRODUCTION}

Turbine performance decreases during the operational life of an aero-engine due to deterioration [1]. For example, abrasion of the rotor-shroud seals can occur during thermal and mechanical load transients [2] and during hard-landings. Larger seal clearances allow more over-shroud leakage flow. This not only decreases the performance of the stage in which it occurs but also, when the leakage flow is re-introduced back into the mainstream, it can adversely affect the aerodynamics of a downstream stage.

In many high bypass ratio civil aero-engines a shrouded multi-stage Low-Pressure Turbine (LPT) is used to power the fan which produces the majority of the engine's thrust. Using the analysis presented in [3], a 1\% decrease in LPT efficiency due to deterioration increases the specific fuel consumption by $0.6 \%$ for an aero-engine with a bypass ratio of six. This increases the annual fuel cost by approximately $\$ 70,000$ per aircraft.

Denton [4] summarised the key aerodynamic features of the leakage flow over shrouded turbine rotor blades. It is a pressure driven leakage which reduces the mass flow rate through the rotor passage, leading to a reduction in the work output. The leakage flow experiences very little change in tangential velocity as it passes over the rotor shroud. Hence, when it re-enters the mainstream, downstream of the rotor blade, it has a higher tangential velocity than the flow leaving the rotor passage.

Depending on the geometry of the rotor over-tip cavity and the distance to a downstream stator the re-introduction of the leakage flow into the mainstream could be a combination of two processes. One extreme would be that the leakage flow mixes with the mainstream immediately downstream of the rotor thereby generating entropy which would probably be considered as additional loss attributed to that turbine stage. Another extreme would be that the leakage flow approaches the casing endwall region of the downstream stator as an axisymmetric jet at a large negative incidence, more than $60^{\circ}$, and the mixing 
occurs within the downstream blade rows generating entropy which is attributed to the downstream stage.

Many of the multi-stage experimental facilities, for example $[5,6]$, used in previous studies of rotor shroud leakage had sharp cornered exit cavities such that the leakage flow re-enters the mainstream with a large radial velocity component leading to strong mixing; this corresponds to the first extreme discussed above. The experimental facility used in this study is more representative of real aero-engine geometry because the exit cavity has a chamfer (shown later in Fig. 2) which guides the leakage flow smoothly back into the mainstream; this corresponds more closely to the second extreme discussed above. Therefore it is appropriate to revisit the study of rotor over-shroud leakage flow for a case where little mixing is expected to occur ahead of any downstream blade row.

Aero-engine designers have striven to keep the performance detriment of the leakage flow as small as possible by minimising the clearance over the rotor shroud knife-edge seals and adding incidence tolerance to the downstream stator. However, inservice deterioration increases the rotor clearance and several researchers, for example [5, 7], have found that increasing the leakage mass flow rate leads to intensification and radial migration of the secondary flow in the downstream stator.

A variety of rotor shroud leakage path redesigns have been investigated in the literature, including modifications to the way in which the leakage flow re-enters the mainstream [8, 9] and flow control methods [10]. Several studies have investigated the possibility of turning the leakage flow towards the mainstream flow direction before the leakage flow re-enters the mainstream, either with bladelets on the rotor shroud or turning vanes on the casing. The bladelets have the advantage of extracting some useful work from the leakage flow. However, Wallis et al. [6] found that the benefits of bladelets were outweighed by the reduced effectiveness of the leakage flow in preventing mainstream flow from entering the shroud exit cavity, which then re-entered the mainstream as highly swirling flow, leading to increased losses. Rosic and Denton [11] had some success with turning vanes on the casing endwall; they found that the installation of eight turning vanes almost completely eliminated the high swirl of the leakage flow and increased the stage efficiency by $0.4 \%$. However, they did not investigate the robustness of the design to increases in clearance. Numerical calculations by Gao et al. [12] suggested that the turning vane design is not robust to increases in clearance and would become less effective over the operational life of the turbine.

In this publication it will be shown that the angle of the rotor over-shroud leakage flow is, essentially, independent of both the rotor seal clearance and the turbine operating point (flow coefficient). Consequently, if there is little mixing between the leakage and mainstream flows, the downstream stator does not need large incidence tolerance if it is locally redesigned in the endwall region to match the constant angle of the approaching leakage flow. This eliminates any unnecessary turning of the rotor over-shroud leakage flow thus reducing the entropy generation rate and thereby improving the efficiency of the downstream stages.
This paper is split into five sections. In the first section, the turbine facility, experimental methodology, measurement techniques and computational methods are outlined. In the second section, the aerodynamic performance of the Datum Stator 2 is investigated for different levels of Rotor 1 shroud seal deterioration and the data compared with an extended version of Denton's over tip leakage analysis [4]. In the third section, an extensive computational investigation is undertaken to understand the key fluid dynamics associated with leakage flow entering a downstream stator. In the fourth and fifth sections, a New Stator 2 is designed and then its performance improvement is experimentally verified.

\section{METHODOLOGY}

Experimental facility: The experimental measurements for the present study have been undertaken using the Peregrine Turbine Facility in the Whittle Laboratory. The Peregrine is a large scale, low-speed, two-stage turbine with high aspect ratio blading with representative rotor over-shroud and stator underhub leakage paths and cavities. The aerodynamic design parameters for the facility are given in Table 1 . The first stage stator (Stator 1) has an axial inlet flow angle, whilst the second stage stator (Stator 2) is representative of an embedded blade row. The first and second stage rotor rows (Rotor 1 and Rotor 2) are identical. The blade loading distribution of the second stage stator and the rotor rows are typical of current aero-engine technology for low pressure turbines. The Datum blading used in this investigation had been designed for an investigation of clocking [13] so the stator blade stacking had been chosen to align the wakes across the span and no endwall geometry features had been included.

Table 1: Aerodynamic parameters of the two-stage low pressure turbine configuration used for the current study

\begin{tabular}{lc}
\hline Number of stages & 2 \\
Mean radius & $0.6477 \mathrm{~m}$ \\
Hub to tip ratio & 0.7 \\
Design flow coefficient (both stages) & 0.822 \\
Design Reynolds number & 148700 \\
Design stage reaction (both stages) & 0.52 \\
Design stage loading (both stages) & 2.02 \\
Nominal blade Mach number & 0.076 \\
Nominal non-dimensional blade speed & 0.0485 \\
Nominal rotational speed & $379 \mathrm{rpm}^{3}$ \\
Nominal volumetric flow rate & $19.7 \mathrm{~m}^{3}{ }^{-1}$ \\
Number of blades: stator, rotor (both stages) & 84,112 \\
Stator exit flow angle (mid-span) & $61.12^{\circ}$ \\
Rotor exit relative flow angle (mid-span) & $-61.75^{\circ}$ \\
Stator axial chord (mid-span) & $50 \mathrm{~mm}^{\circ}$ \\
Rotor axial chord (mid-span) & $35 \mathrm{~mm}$ \\
\hline
\end{tabular}

The Peregrine facility has two controls: the motor and the eddy-current brake. The variable speed motor drives the centrifugal fan, at the rear of the facility, which draws ambient air through the working section. This effectively sets the mass 
flow rate through the facility. The eddy-current brake absorbs the power generated by the turbine. The rotational speed of the downstream fan and the torque applied by the eddy current brake are controlled in real time to maintain the required Reynolds number and flow coefficient. In any one-second interval the fractional noise (expressed as $\sigma / \mu$ ) in the Reynolds number and flow coefficient are $0.04 \%$ and $0.05 \%$ respectively. Further details of the facility and control system are given in [14].

A schematic of the working section of the Peregrine Facility is shown in Fig. 1. The facility has a total of seven measurement planes (MP1 to MP7) at which area traverses can be undertaken. Far upstream of the working section there is a turbulence grid (designed to produce a turbulence intensity of approximately $4 \%)$ to represent the inlet turbulence of an embedded stage. At the reference plane (REF) there are seven mid-span Kiel probes and a mid-span thermocouple. During this investigation two small mid-span Kiel probes, positioned $60^{\circ}$ either side of the traverse location, were fitted at stage 2 inlet (MP4). These were used to investigate the possible benefits of using an inter-stage reference stagnation pressure when determining the Stator 2 entropy loss coefficient.

Experimental methodology: The working section of the turbine facility is contained within five steel casing rings. The first casing ring, designated rotor 0 , does not contain a blade row but the other four do. The Rotor 1 casing ring, shown in Fig. 2, forms the cavity above the shrouded rotor and contains a recess for a tooling board insert. It also contains the probe insertion holes for the MP3 and MP4 traverse planes and has a chamfered exit portion which is representative of the leakage path in an aero-engine. The Rotor 2 casing ring contains the MP5 and MP6 traverse planes, has a similar chamfer but no tooling board insert.

The Stator 2 inlet traverse plane (MP4) passes through the chamfered portion of the Rotor 1 over-shroud cavity. This allows five-hole probe measurements up to $102 \%$ span. The $100 \%$ spanwise position, indicated by the blue dashed line in Fig. 2, corresponds to the casing endwall of the stators and the underside of the rotor shrouds.

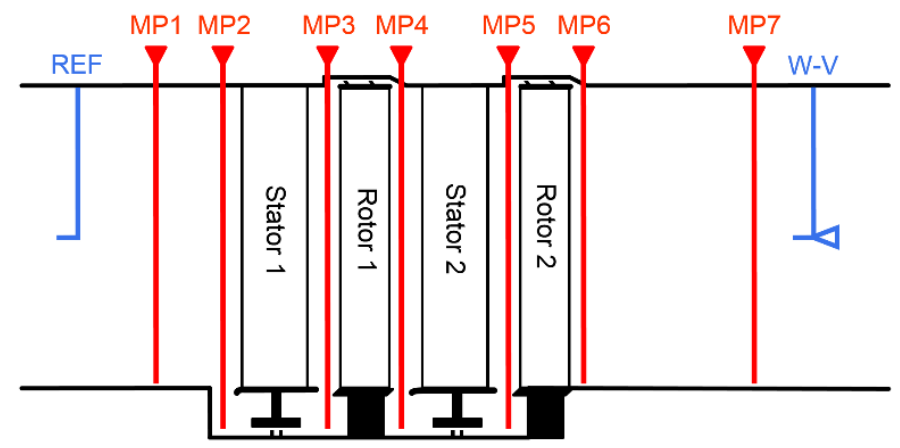

Fig. 1: A schematic of the working section of the experimental facility (meridional view).

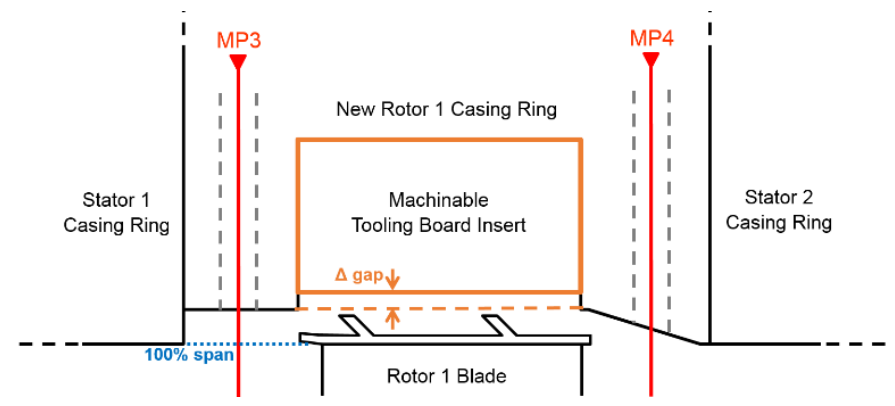

Fig. 2: A schematic of the Rotor 1 casing ring with tooling board insert. The axial locations of MP3 and MP4 are shown.

By machining the tooling board insert the clearance above the Rotor 1 shroud knife-edge seals can be increased, as indicated by the orange arrows in Fig. 2. The Peregrine design clearance is indicated by the dashed orange line in Fig. 2. In this paper the total distance between the rotor shroud knife-edge seal and the tooling board insert is known as the clearance "gap". The non-dimensional deterioration is defined as:

$$
d=\Delta \text { gap } / \text { span }
$$

where $\Delta g a p$ is the total gap minus the Peregrine design value. A non-dimensional deterioration of zero refers to the Peregrine design clearance which corresponds to that of an LPT in a new aero-engine. Four values of the non-dimensional deterioration ranging from zero (new-condition) to a well-worn (oldcondition) aero-engine were investigated (see Table 2).

Table 2: Values of deterioration (the increase in Rotor 1 clearance gap above the rotor shroud) investigated (nondimensional deterioration $\boldsymbol{d}=\Delta \mathrm{gap} / \mathrm{span}$ ).

\begin{tabular}{lllll}
\hline Ugap $(\mathrm{mm})$ & 0.0 & 0.6 & 1.4 & 4.0 \\
$d(\%)$ & 0.00 & 0.26 & 0.61 & 1.75 \\
\hline
\end{tabular}

Measurement techniques: All of the experimental data presented in this paper are area traverse measurements at planes MP4, MP5 and MP6 using a calibrated conical-head, five-hole probe. Taylor and Longley [15] reported that a similar five-hole probe when used in a cascade produced repeatable measurements to within $\pm 0.15 \%$ of the dynamic head. This corresponds to an entropy loss coefficient within the current facility of \pm 0.0007 . Wall proximity studies suggested that the measurements would be compromised when the probe head was within one head diameter of the wall: such points were discarded.

Effect of Probe Insertion: During experimentation it was observed that the insertion of the five-hole probe at measurement planes upstream of a rotor row (MP3 or MP5) had a measurable impact of the performance of the turbine; however when inserted downstream of a rotor row (MP4 or MP6) it did not. This effect was verified during an area traverse at Rotor 2 inlet (MP5) by comparing the auto-correlation of the variation in the turbine work (Fig. 3a) with the auto-correlation of the probe spanwise position (Fig. 3b). The auto-correlation of the turbine work is a maximum every time the probe is fully withdrawn. (To avoid 
backlash, measurements are only taken when the probe is moved radially inward.)

The discrete Fourier transform can be used to estimate the magnitude of the probe-stem effect. Based on 20 repeat MP5 area traverses, the average peak-to-peak effect of fully inserting the probe at MP5 was $0.063 \%$ on turbine work and $0.071 \%$ on total-to-total efficiency.

The probe stem effect is small and only exists when the probe is inserted upstream of a rotor row (probably associated with the stem blockage affecting the axial-flux of tangential momentum). It therefore contributes a small fixed offset when assessing the entropy generation rate across a blade row.

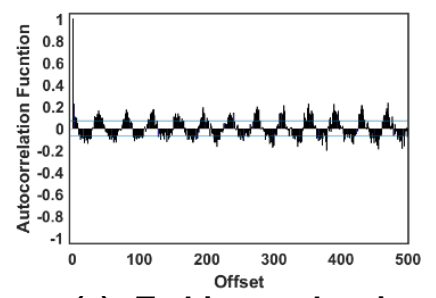

(a) Turbine work noise

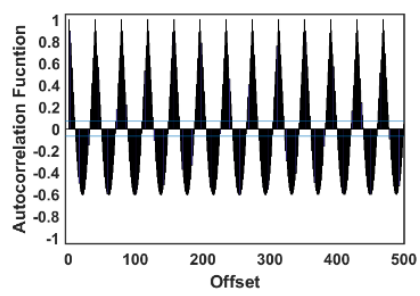

(b) Spanwise position
Fig.3: Autocorrelation function against offset for turbine work noise and the spanwise position of the probe for an area traverse at Rotor 2 inlet (MP5).

Accounting Methodology: Previous investigations using the Peregrine facility found that its performance is affected by five independent non-dimensional groups [16]. Two of these groups, the Reynolds number and the flow coefficient, are controlled by the facility control system. There are three more nondimensional groups which are affected by ambient conditions (humidity, ambient and rig temperatures) but are uncontrolled. These are: the non-dimensional blade speed, $b$, the nondimensional clearance, $c$, and the gas expansions $g$ :

$$
b=\frac{U}{\sqrt{c_{p} T_{0, \text { ref }}}}, \quad c=\frac{\text { gap }}{\text { span }} \quad \text { and } \quad g=\frac{c_{p}}{R}
$$

In previous experimental work using the Peregrine facility, the problem of uncontrolled effects due to changes in ambient conditions was combated using the best-fit accounting methodology developed by Evans and Longley [16].

Stator 2 entropy generation rate: For a fixed second stage work output, the key quantity of interest in determining the efficiency is the entropy generation rate across Stator 2 (and Rotor 2). This is quantified using the entropy loss coefficient:

$$
\zeta=\frac{T_{0, r e f} \Delta s}{U^{2}}
$$

where $U$ is the mean blade speed. In the above the mass average entropy change, $\Delta s$, is determined from the change in the mass average stagnation pressure ${ }^{1}$ along a streamtube between an area traverse at Stator 2 exit (MP5) and one at Stator 2 inlet (MP4). Each of these area traverses is measured twice and so there are

${ }^{1}$ Although the stagnation enthalpy is non-uniform within Stator 2, energy conservation eliminates its effect from the entropy generation rate. four possible estimates for the entropy loss coefficient. The spread between the maximum and minimum estimate, referred to as the peak-to-peak variation, is an indicator of the consistency of the experimentation.

As-measured data: The maximum and minimum estimates for the entropy loss coefficient for the entire mass flow through the Datum Stator 2 is shown in Fig. 4 (blue diamonds) for the four values of non-dimensional deterioration. Although it is clear that the deterioration increases the entropy loss coefficient the spread in the data is quite large.

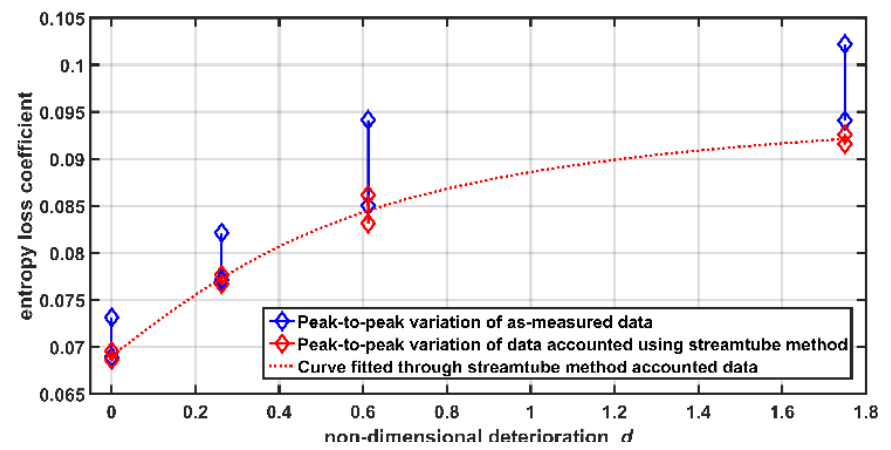

Fig. 4: Measured full span entropy loss coefficient across Datum Stator 2 using as-measured data and streamtube accounting method (accounted using $30-40 \%$ streamtube).

For the four values of deterioration the average spread in the Stator 2 as-measured entropy loss coefficient is approximately fifteen times larger than the expected repeatability of the fivehole probe measurements (see Table 3). For each traverse, the mass average stagnation pressure is measured relative to the turbine inlet reference stagnation pressure. If there is any change in the uncontrolled non-dimensional groups between when the Stator 2 inlet and exit traverses are undertaken the stagnation pressure drop across the first stage (about six working section dynamic heads) is affected and so determining the small stagnation pressure drop due viscous effects within Stator 2 is compromised. Therefore, the observed large spread in the entropy loss coefficient is the result of changes in the uncontrolled non-dimensional groups between individual runs.

One method to eliminate these changes in the first stage performance is to measure the traverses relative to the mid-span stagnation pressure at inlet to Stator 2 using the inter-stage Kiel probes. Although this reduced the spread by approximately a factor of two, it was still seven times larger than the expected repeatability of the five-hole probe measurements (see Table 3).

The original accounting methodology [16] used a best-fit approach to eliminate the effects of uncontrolled nondimensional groups from a large family of closely related clocking measurements. That approach is not convenient here so an alternate method for accounting for the uncontrolled changes in the first stage performance was investigated. 
The Streamtube Accounting Method: All of the Rotor 1 overshroud leakage flow must re-enter the mainstream between the Rotor 1 and Stator 2. Therefore a reasonable assumption is that the effects of the leakage flow are localised to the outer portion of the span. Hence, the effects of increasing the amount of overshroud leakage would be expected to diminish towards the inner portion of the span. For the streamtube accounting method it is assumed that changing the amount of the Rotor 1 shroud-leakage flow has no effect on the entropy generation rate across Stator 2 (or Rotor 2) within a specified streamtube in the inner span.

For the current investigation, the accounting streamtube chosen corresponded to $30-40 \%$ mass flow which is above the hub endwall secondary flow. Hence, to determine the entropy generation rate across the Stator 2 (or Rotor 2) the mean value of the stagnation pressure at the traverse planes were adjusted so that the $30-40 \%$ mass flow streamtube always has the same value of the entropy loss coefficient (which was arbitrarily set using the datum geometry).

Table 3: Comparison of the average spread across the four deteriorations in determining the Datum Stator 2 entropy loss coefficient.

\begin{tabular}{ll}
\hline As-measured (upstream reference) & 0.0100 \\
As-measured (inter-stage Kiel reference) & 0.0050 \\
Streamtube accounting method (30-40\%) & 0.0016 \\
Five-hole probe repeatability & 0.0007 \\
\hline
\end{tabular}

The entropy loss coefficient for the Datum Stator 2 determined using the streamtube accounting method is shown in Fig. 4 (red diamonds, using the $30-40 \%$ streamtube). The streamtube accounting method has reduced the average spread in determining the entropy loss coefficient by approximately a factor of six compared to the as measured data, see Table 3 . It is worth noting that the streamtube accounting method has reduced the average spread in determining the entropy loss coefficient to approximately twice the five-hole probe repeatability.

An empirical curve-fit through the entropy loss coefficient using the streamtube accounting method is also shown on Fig. 4 (red dotted line). It is unclear why the streamtube method follows the minimum estimates but it does mean that any predicted performance gains might actually be larger in practice.

The robustness of the streamtube accounting method, and the selection of the $30-40 \%$ mass flow accounting streamtube, can be assessed by examining the entropy generation rate in the adjacent streamtubes (20-30\% and $40-50 \%$ ) for different values of non-dimensional deterioration. These are shown in in Fig. 5 along with the curves for the full-span (0-100\%) and the tip region (70-100\%). Looking at the entropy loss coefficients for the streamtubes $20-30 \%$ and $40-50 \%$, which are adjacent to the accounting streamtube, there is very little variation with nondimensional deterioration. This supports the streamtube accounting method which will be used throughout the rest of this paper to determine the entropy loss coefficient.

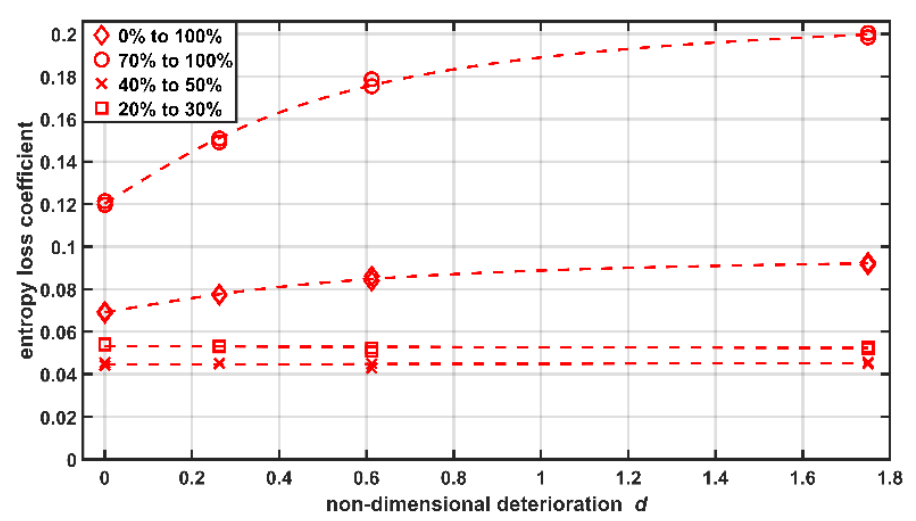

Fig. 5: Measured Datum Stator 2 Entropy loss coefficient for 0-100\%, $70-100 \%, 40-50 \%$ and $20-30 \%$ mass flow, accounted using the $30-40 \%$ streamtube method.

Computational method: A computational investigation was used to understand the fluid dynamic mechanisms and to support the re-design approach. All of the computational studies presented here have been obtained using the steady state nonlinear version of HYDRA, which is the Rolls-Royce in-house code. HYDRA is a general purpose flow solver for hybrid unstructured meshes which uses an edge-based data structure. For further details of the code see [17]. Although the current geometry is representative of a low pressure turbine, where the blade boundary layers would be expected to be primarily laminar, the major focus is on the endwall region and the interaction between the shroud leakage flow and the downstream stator. Therefore, for convenience, all calculations were run fully turbulent using the Spalart-Allmaras (S-A) turbulence model (no transition model). However, it is noted that S-A model gives a very low turbulent viscosity at low Reynolds number.

To quickly investigate the effect of the different Rotor 1 leakage flows on Stator 2, just the flow field within stage 2 was calculated. The axial position of the domain inlet corresponds to the Stator 2 inlet (MP4). The circumferentially-averaged spanwise profiles of stagnation pressure, yaw angle and pitch angle measured at MP4 were used for the inlet boundary conditions for each value of the non-dimensional deterioration. The inlet S-A viscosity profile was extracted from a previous two-stage steady state calculation of the Peregrine rig at MP4 [18]. For all of the calculations the domain exit static pressure was adjusted so that the area-averaged axial velocity at the inlet was within $0.01 \%$ of the design condition. The exit domain is 1.8 span-lengths downstream of MP6 to decouple the radial equilibrium of the flow within the rotor from the exit boundary condition. The detail of the Rotor 1 shroud cavity exit chamfer is included, but all other endwalls are at a constant radius.

\section{DATUM STATOR 2 WITH ROTOR 1 DETERIORATION}

Datum Stator 2 inlet flow field: The spanwise plots of the flow conditions between $85-102 \%$ span at Stator 2 inlet (MP4) for the four values of the non-dimensional deterioration are 


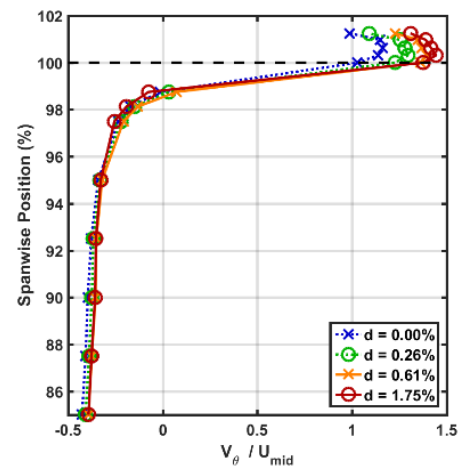

(a) Tangential Velocity

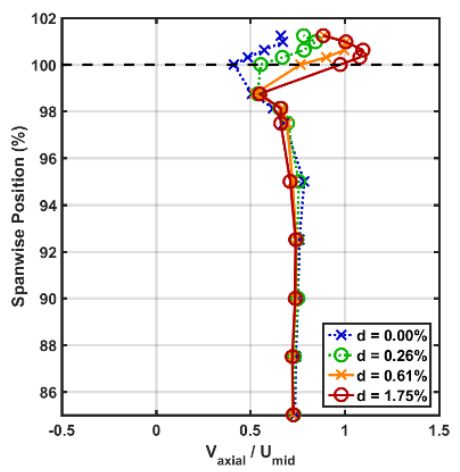

(b) Axial Velocity

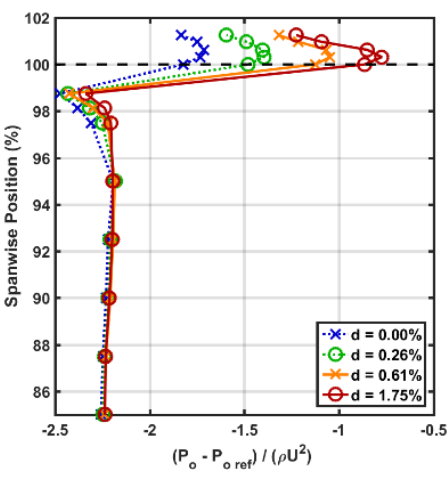

(c) Stagnation Pressure

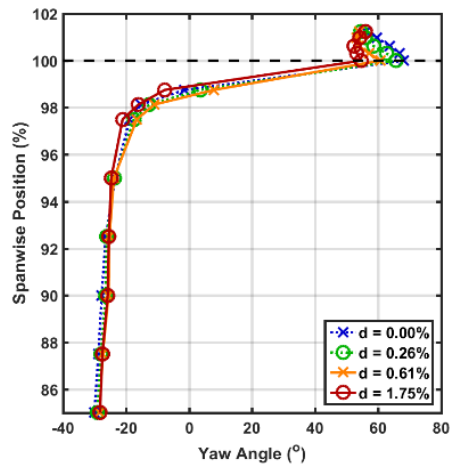

(d) Yaw Angle

Fig. 6: Spanwise plots of the flow conditions from 85-102\% span at Datum Stator 2 inlet (MP4) for four values of nondimensional deterioration, $\boldsymbol{d}$. The dashed black line indicates $100 \%$ span. Points above $100 \%$ are within the exit portion of the Rotor 1 shroud cavity.

shown in Fig. 6. Increasing the clearance between the Rotor 1 knife-edge seal and the casing is expected to increase the overshroud leakage mass flow rate and there will be a corresponding decrease in the mass flow rate through the Rotor 1 passage [19].

Tangential velocity: The pitchwise mass-averaged, nondimensional tangential velocity profiles measured are shown in Fig. 6a. The tangential velocity in the exit cavity is much higher than that of the mainstream: the magnitude is approximately three times larger and it is in the opposite direction. This is because the tangential momentum of the leakage flow is approximately conserved through the leakage path [4].

The tangential velocity of the over-shroud leakage flow increases with the non-dimensional deterioration. This is a combination of two effects. Firstly, increasing the shroud leakage mass flow rate means more of the Stator 1 casing endwall flow is ingested into the shroud cavity upstream of Rotor 1 . Thus the tangential velocity, and stagnation pressure, in that cavity would be expected to increase. Secondly, as the non-dimensional deterioration increases the width of the leakage jet increases such that a smaller proportion of the flow is effected by the dissipation in the shear layers at the edge of the jet.

The strong gradient in the tangential velocity between $99 \%$ and $100 \%$ span indicates that for this aero-engine representative geometry there is minimal mixing between the leakage and mainstream flows upstream of Stator 2 (MP4). Making use of this observation, the flow above and below the $100 \%$ span line will be referred to as the leakage flow and the mainstream flow, respectively.

Axial velocity: As the clearance gap above the Rotor 1 shroud increases, the mass flow rate of the over-shroud leakage flow increases. However, the area of the exit cavity at the axial plane where the measurements are taken is fixed. Therefore, as shown in Fig. 6b, the axial velocity measured in the exit cavity increases as the non-dimensional deterioration increases.

Stagnation pressure: The pitchwise mass-averaged, nondimensional stagnation pressure profiles measured at the inlet to the Datum Stator 2 (MP4) are shown in Fig. 6c. The stagnation pressure of the over-shroud leakage flow is higher than that of the mainstream flow. This shows that the viscous dissipation within the shroud leakage flow is smaller than the stagnation pressure drop of the mainstream flow due to work extraction.

When the non-dimensional deterioration is increased there are two effects which raise the stagnation pressure of the shroud leakage flow in the exit cavity. Firstly, the stagnation pressure in the upstream cavity is expected to rise as more of the Stator 1 endwall flow passes above the rotor shroud. Secondly, as the width of the leakage jet increases, a smaller proportion of the kinetic energy is dissipated in the shear layers at edges of the jets.

By integrating over the exit cavity region, it is found that the mass-averaged stagnation pressure of the over-shroud leakage flow increases by 2.4 working section dynamic heads $\left(\frac{1}{2} \rho v_{\text {axial }}^{2}\right)$ between the smallest and largest deteriorations. The stagnation pressure of the mainstream flow reduces by approximately 6.6 working section dynamic heads due to work extraction. At large values of deterioration there is a slight increase in the stagnation pressure of the mainstream flow due to reduced work extraction, which is consistent with [19]. Between 96-99\% span the casing endwall secondary flow within Rotor 1 is reduced as more of the Stator 1 endwall flow passes over the rotor shroud.

Yaw angle: The measured pitchwise averaged yaw angle profiles are shown in Fig. 6d. The yaw angle of the leakage flow is approximately $90^{\circ}$ higher than that of the mainstream. The yaw angle in the mainstream is approximately $-30^{\circ}$ and is set by the rotor trailing edge angle and the flow coefficient, whereas, the yaw angle of the leakage jet is approximately $60^{\circ}$.

Over-shroud tip leakage model: Denton [4] made three assumptions about the leakage flow over shrouded turbine rotors: inviscid flow, conservation of tangential momentum through the leakage path and uniform static pressure in the shroud exit cavity (i.e. the leakage jet is fully expanded after the final knife-edge seal). Combining these assumptions with a chamfered re-entry of the leakage flow into the mainstream, an inviscid model can be developed (see Appendix) to estimate the yaw angle of the leakage flow downstream of Rotor 1 : 


$$
\tan \left(\alpha_{\text {leakage }, M P 4}\right)=\frac{\tan \alpha_{M P 3}}{\cos \gamma \sqrt{\left(\frac{1}{\cos \alpha_{M P 4}^{\text {rel }}}\right)^{2}-\left(\tan \alpha_{M P 3}-\frac{1}{\phi}\right)^{2}}}
$$

From the above, it can be seen that for inviscid flow the yaw angle of the leakage flow downstream of Rotor 1 (MP4) is independent of the non-dimensional deterioration and is only a weak function of the flow coefficient, $\phi$. This was experimentally verified by undertaking area traverses at MP4 for a range of flow coefficients corresponding to $\pm 3^{\circ}$ incidence of the mainstream on to Stator 2. There was no observable change to the yaw angle of the shroud leakage flow across this range.

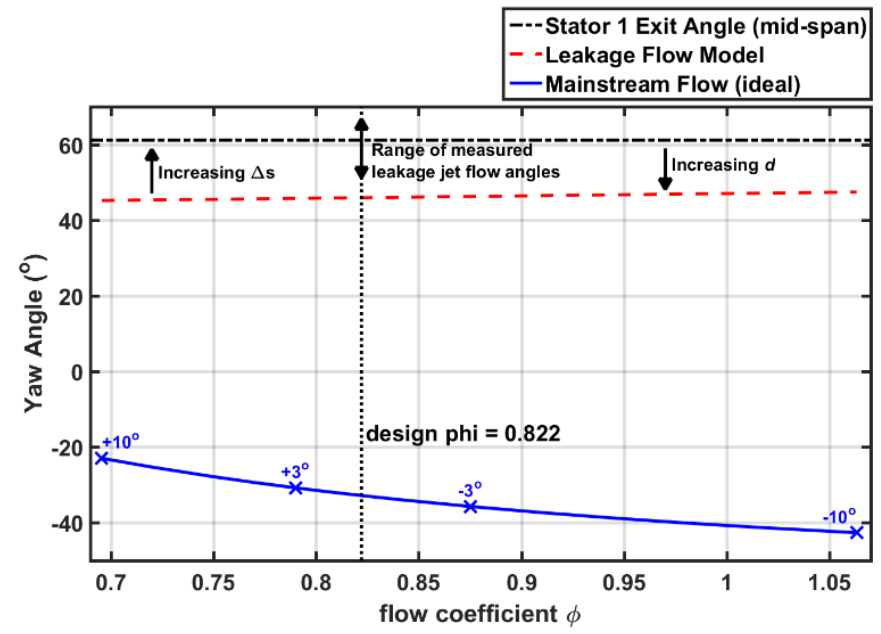

Fig.7: The inviscid yaw angle of the leakage flow and the mainstream flow downstream of Rotor 1 (MP4) against a range of flow coefficients corresponding to change of incidence of $\pm 10^{\circ}$ in the mainstream.

The inviscid model for the yaw angle of the leakage flow and the ideal mainstream yaw angle downstream of Rotor 1 (MP4) are plotted in Fig. 7. The range of flow coefficients plotted corresponds to a change of incidence of $\pm 10^{\circ}$ in the mainstream. The leakage flow inviscid model predicts a peak-to-peak variation of $2.3^{\circ}$ across the range of flow coefficients shown.

The model has assumed inviscid flow, but in reality there will be some viscous dissipation within the leakage path. If the assumption of inviscid flow is relaxed, but the other two of Denton's assumptions hold, then the meridional velocity of the leakage jet will reduce and the yaw angle of the leakage jet will increase, as indicated on Fig. 7. The range of measured yaw angles of the leakage flow are also indicated. The measured values are higher than the value predicted by the inviscid model, as expected due to viscous dissipation in the leakage path.

As the non-dimensional deterioration increases the stagnation pressure of the leakage flow downstream of Rotor 1 (MP4) increases (see Fig. 6c), and the yaw angle of the leakage jet reduces from $65^{\circ}$ to $53^{\circ}$ (see Fig. 6d). This is towards the value predicted by the inviscid model, as indicated on Fig. 7.

The above observations appear to contradict previous studies such as those of [5] and [7] which found that the spanwise extent and magnitude of the negative incidence onto the downstream stator increased as the shroud leakage mass flowrate increased. However, for both of those previous studies the exit cavities had sharp corners such that the leakage flow entered the mainstream with a large radial velocity component leading to strong mixing with the mainstream flow. This corresponds to the first extreme case discussed earlier. Further, in both of those experimental facilities the measurement plane was downstream of the exit cavity. The current authors suggest that the yaw angle of the shroud leakage flow was approximately constant in those studies and that the increase in angle and spanwise extent measured by the probe resulted from the strong mixing of leakage with the mainstream ahead of the measuring plane.

In summary for the aero-engine representative geometry studied here, the yaw angle of the leakage flow is a weak function of the flow coefficient and the angle variation with deterioration is small compared to the overall difference between the leakage flow angle and the mainstream flow angle.

Leakage mass flow rate: As previously stated, the strong gradient in tangential velocity between $99 \%$ and $100 \%$ span in Fig. 6a suggests that for the aero-engine representative geometry there is minimal mixing between the leakage and mainstream flows upstream of MP4. Hence, the leakage mass flow rate can be approximated by integrating the axial velocity over the cavity region at MP4. The change in leakage mass fraction, relative to the value at the design clearance, is plotted against the nondimensional deterioration in Fig. 8. The data has been empirically fitted with an inverse tan curve. The leakage mass fraction increases approximately linearly for small values of the non-dimensional deterioration, as predicted by Denton [4], but begins to level off for larger values of the non-dimensional deterioration.

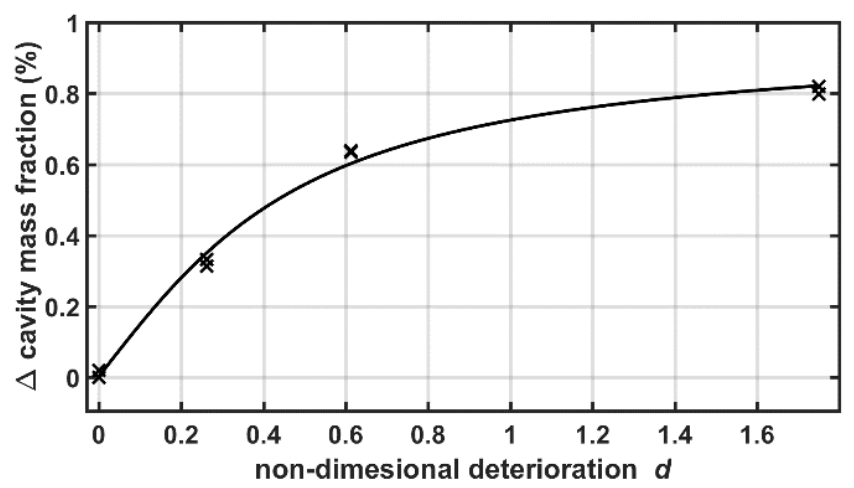

Fig. 8: Measured change in Rotor 1 leakage mass fraction, relative to the value at the design clearance, plotted against the non-dimensional deterioration (data has been empirically fitted with an inverse tan curve).

The outline of the cavity above Rotor 1 is shown in Fig. 2. As the clearance gap increases above the knife-edge seals, the gap becomes comparable in area to other sections of the leakage path, which could start to behave as additional throats. This effect was also observed by Yoon, et al. [19] and explains why the leakage mass fraction does not continue to increase linearly for large values of deterioration. 
Datum stator 2 exit flow field: For the four values of nondimensional deterioration, the contours of the non-dimensional streamwise vorticity at Stator 2 exit (MP5) are shown in Fig. 9. For the non-dimensional deterioration value of $0.00 \%$ a region of positive streamwise vorticity can be seen towards the top of span, on the suction side of the wake. On closer inspection, it can be seen that the region of positive vorticity has two distinct cores. As the non-dimensional deterioration increases, these two vortices become more distinct, spreading apart and growing in strength. For each value of the deterioration there is also a region of strong negative vorticity aligned with the wake and adjacent to the two positive vortices.

The fact that two distinct large positive vortices are visible suggests that there are two distinct mechanisms at play. It will be shown that neither of these vortices result from the standard horseshoe vortex model of secondary flow.

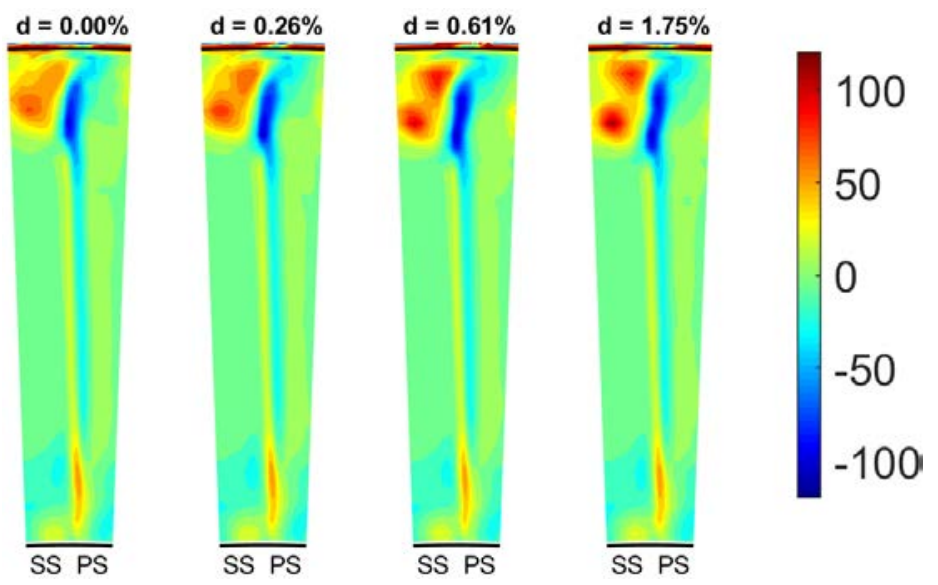

Fig. 9: Measured contours of non-dimensional streamwise vorticity at Datum Stator 2 exit (MP5), for values of nondimensional deterioration.
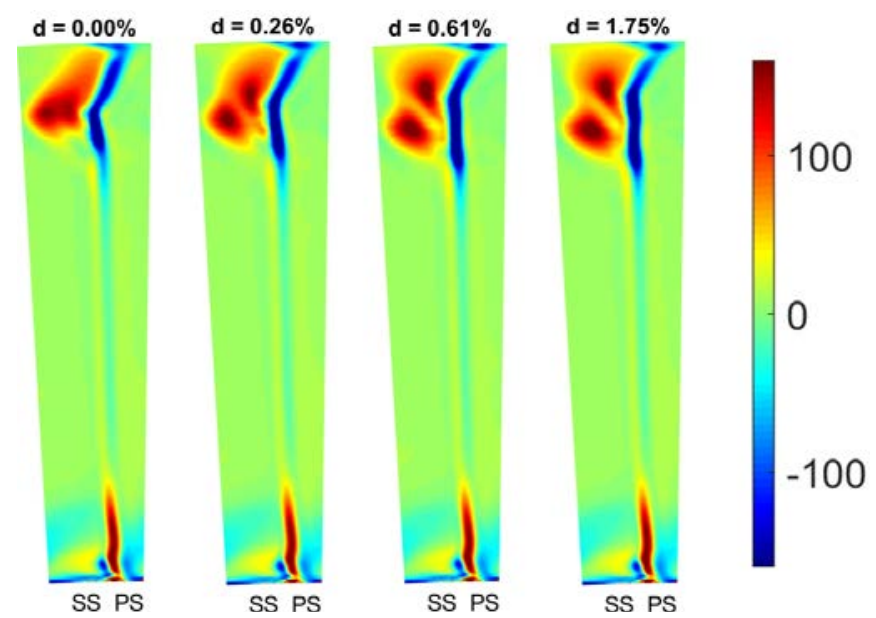

Fig. 10: Calculated contours of the non-dimensional streamwise vorticity at Datum Stator 2 exit (MP5), for values of non-dimensional deterioration.

\section{COMPUTATIONAL INVESTIGATION}

For the four values of non-dimensional deterioration, the calculated contours of the non-dimensional streamwise vorticity at Datum Stator 2 exit are shown in Fig. 10. There is good qualitative agreement with the measured contours which are shown in Fig. 9. The calculations have produced two positive vortices in the casing endwall region. As the non-dimensional deterioration increases the two vortices become stronger and more distinct. For each value of deterioration there is also a region of strong negative vorticity aligned with the wake and adjacent to the two positive vortices.

Fluid dynamic mechanisms: The calculations capture the main features and trends observed in the measurements of the Datum Stator 2 exit flow. The origin of the two positive vortices will now be investigated using the Q-criteria of the calculated flow field. This is an analysis of the velocity gradient tensor which defines a vortex as a "connected region of fluid with a positive second invariant of $\nabla u$ ” [20].

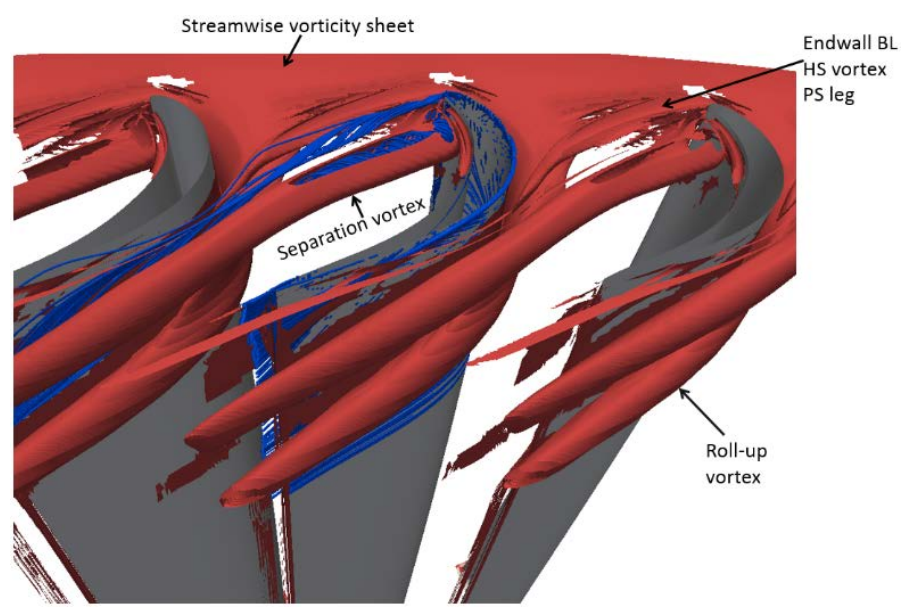

Fig. 11: Calculated contours of Q-criteria $\left(10 \times 10^{6}\right.$ to $20 \times$ $10^{6}$ ) with positive streamwise vorticity within the Datum Stator 2 passage for non-dimensional deterioration value of $1.75 \%$. The blue streamlines were seeded on the blade surface of the middle stator of the three stators shown.

The calculated contours of the Q-criteria within the Stator 2 passage for the non-dimensional deterioration value of $1.75 \%$ are shown in Fig. 11, viewed looking upstream. Looking first at the aft-section of the suction side of the blade it can be seen that the contours of the Q-criteria identify two positive vortices (only contours of Q-criteria with positive streamwise vorticity are shown). These two vortices will be referred to as the separation vortex and the roll-up vortex, as indicated on Fig. 11.

The separation vortex originates near the pressure side leading edge of the stator and sweeps across the passage to the suction side of the adjacent stator. It will be shown that this vortex is caused by the separation of the over-shroud leakage flow off the pressure side of the leading edge.

Following the roll-up vortex back up-stream from the suction side trailing-edge towards the leading edge, it can be seen 
that it remains close to the suction side throughout the passage. At the suction side leading edge the roll-up vortex appears from the band of positive streamwise vorticity which stretches across the stator passage inlet. It will be shown that the roll-up vortex is generated by the roll up of a sheet of streamwise vorticity at the inlet to the stator passage.

A third positive streamwise vortex is also identified by the Q-criteria contours. It originates just upstream of the leading edge and migrates across the passage towards the suction side. This is the pressure side leg of the horseshoe vortex due to the casing endwall boundary layer. This vortex is small in comparison to the other two vortex structures and remains confined to the casing endwall.

The Separation Vortex: The leakage flow meets the Datum Stator 2 leading edge with a high negative incidence and separates off the pressure side of the leading edge, which sets up a recirculation zone behind the separation line. The Datum Stator 2 pressure side boundary layer vorticity, associated with the mainstream blade loading, is shed as a separation vortex at a point of separation on the pressure side of the stator, just downstream of the leading edge. The blue streamlines of Fig. 11 were seeded on the surface of the middle stator of the three stators shown. It can be seen that the pressure side surface flow separates from the surface and rotates within the separation vortex Q-criteria contours. Similar behaviour has been seen in axial compressors [21, 22] on the suction side of the leading edge, which leads to a spike-type route to rotating stall.

Giboni et al. [23] also identified a large recirculation zone along the first quarter of the pressure side of the stator due to the negative incidence of the leakage flow. However, the experimental facilities used in previous rotor shroud leakage investigations had sharp cornered exit cavities, for example [5, 6], such that leakage flow re-enters the mainstream with a strong radial velocity component leading to strong mixing, reducing the incidence onto the downstream stator. However, the current facility has an aero-engine-representative chamfered exit cavity. This may explain why such a dramatic separation vortex has not been described in this context in the literature before.

The pressure side boundary layer contains positive radial vorticity filaments. At the point of separation the vorticity filaments lift off the surface and are turned towards the streamwise direction, resulting in a separation vortex with positive streamwise vorticity. At spanwise positions above the separation point the freestream exhibits flow reversal due to the large separation. Hence, in this region close to the endwall on the pressure side the boundary layer contains negative radial vorticity filaments. These are also turned towards the streamwise direction at the separation point thus producing positive streamwise vorticity, as illustrated in Fig. 12.

The point at which a three-dimensional separation occurs can be determined by examining the skin-friction lines on the blade surface. Any point on the surface can only be crossed by a single skin-friction line, except at a critical point. A critical nodal point is a point common to an infinite number of limiting streamlines, where both orthogonal shear stress vectors $\tau_{x}$ and $\tau_{y}$ are identically zero [24]. A close-up of the pressure side leading edge for the non-dimensional deterioration value of $1.75 \%$ is shown in Fig. 13. The surface skin friction lines are shown in black. The blue streamtubes were seeded on the blade surface. It can be seen that the separation vortex is shed from the surface at a nodal critical point on the pressure side surface, just downstream of the leading edge. The leakage flow separates along a separation line on the leading edge of the stator, which cannot be seen in the figure due to the viewing angle.

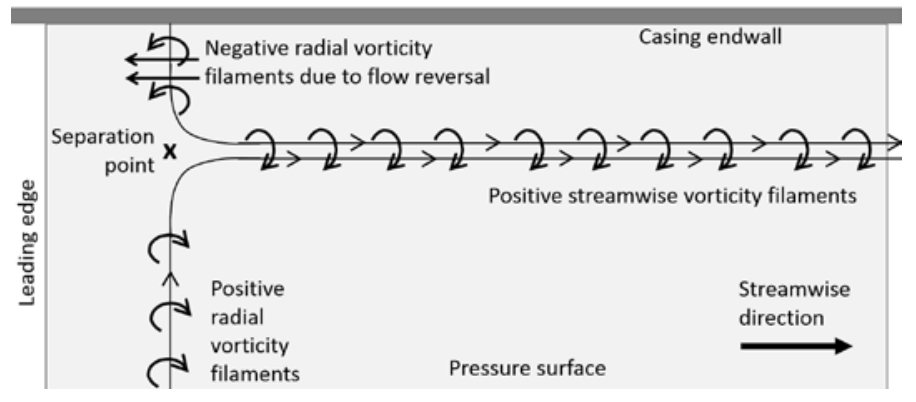

Fig. 12: A two-dimensional diagram to illustrate how the pressure side boundary layer radial vorticity filaments are turned towards the positive streamwise direction at the separation point.

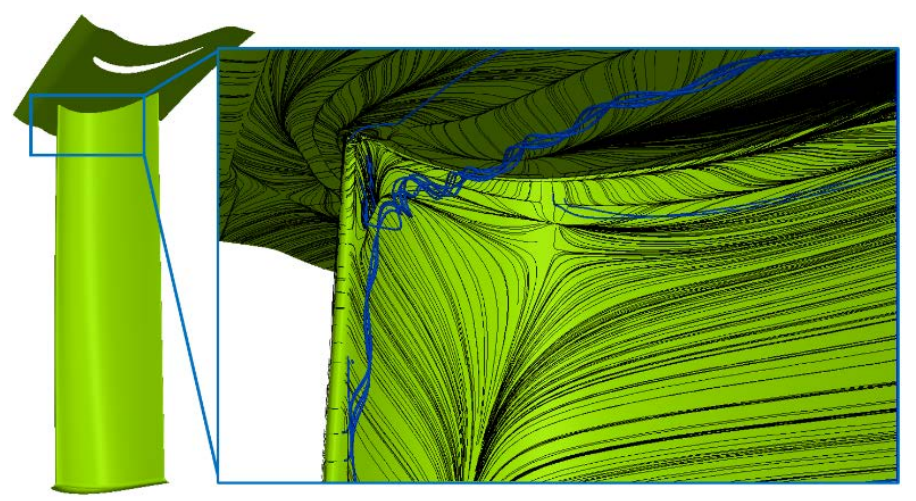

Fig. 13: Close-up of Datum Stator 2 pressure side leading edge for non-dimensional deterioration value of $1.75 \%$. The calculated surface skin friction lines are shown in black. The blue streamtubes were seeded on the blade surface.

The separation vortex sweeps across the passage from the pressure side to the suction side, behaving much like a large passage vortex. Separation lines are visible in the skin friction lines on the casing in Fig. 13, which indicate that the casing boundary layer is swept up into the separation vortex.

The initial vorticity within the separation vortex is originally generated within the blade surface boundary layers, ie within the passage. The separation vortex is purely a result of the Rotor 1 leakage flow approaching the Datum Stator 2 leading edge with high negative incidence. Hence, the separation vortex might be eliminated by redesigning the stator blade in the casing endwall region to reduce the incidence onto the stator.

The Roll-up Vortex: The strong gradient in tangential velocity between the mainstream flow and the Rotor 1 shroud leakage flow results in an approximately axisymmetric sheet of 
concentrated streamwise vorticity entering Stator 2. In isolation this sheet is quasi-stable but once perturbed will breakdown into discrete vortices [25]. When the streamwise vorticity sheet enters the Stator 2 passage the image vortex system causes it to be distorted - it is swept down the suction surface and up the pressure surface. A parametric computational investigation was undertaken which confirmed that a sheet of streamwise vorticity does undergo a Kelvin-Helmholtz style instability and rolls up into a single concentrated vortex (see [26] for details). Crucially, the streamwise vorticity enters at the inlet to the passage and hence, cannot be eliminated within the stator. The roll-up vortex is a fundamental consequence of the Rotor 1 leakage flow.

\section{ELIMINATING SEPARATION VORTEX: NEW STATOR 2}

Design methodology: It was shown previously that the yaw angle of the leakage flow is approximately $90^{\circ}$ higher than that of the mainstream. The three aims of the New Stator design were:

- $\quad$ reduce the incidence by re-cambering the leading edge;

- $\quad$ keep the trailing edge angle unchanged;

- $\quad$ keep the throat width unchanged.

The philosophy behind the design methodology was that unnecessary turning of the Rotor 1 over-shroud leakage flow will lead to unnecessary entropy generation. Furthermore, the flow angle of the leakage jet is independent of the flow coefficient and only varied by $10^{\circ}$ when the non-dimensional deterioration was increased from $0.00 \%$ to $1.75 \%$. Hence, increasing the incidence tolerance by thickening the endwall region of the stator is unnecessary (especially when thicker blades will increase both the velocity outside the boundary layer and the diffusion on the rear portion of the blade). Instead, the inlet angle of the stator was turned towards the direction of the Rotor 1 shroud leakage flow in the casing endwall region.

A number of different inlet angles were numerically investigated, as well as a range of spanwise extents over which the change of inlet angle was applied. The conclusion of the study was that the design methodology is robust: small changes to the chosen inlet angle, or to the spanwise position at which the change of inlet angle begins, do not have a large impact on the Stator 2 performance. The inlet angle of $20^{\circ}$ was chosen as a compromise between predicted performance and machinability.

Profiles of the New Stator 2 at 90\%, 96\% and 99\% span are shown in Fig. 14. At 99\% span the inlet angle has been increased from $-33^{\circ}$ (datum) to $20^{\circ}$. At $90 \%$ span the stator has the datum profile, the profile at $96 \%$ is an intermediate profile. Comparing the three curves at the trailing edge, it can be seen that the trailing edge is unchanged for the new stator profiles. The aft profile of the suction side is also identical, hence the throat width is unchanged, as required.

The leading edge angle begins to change above 93\% span. In order to ensure a smooth change of inlet angle, a shapepreserving piece-wise cubic interpolation was used to generate the intermediate profiles between $93 \%$ and $100 \%$.

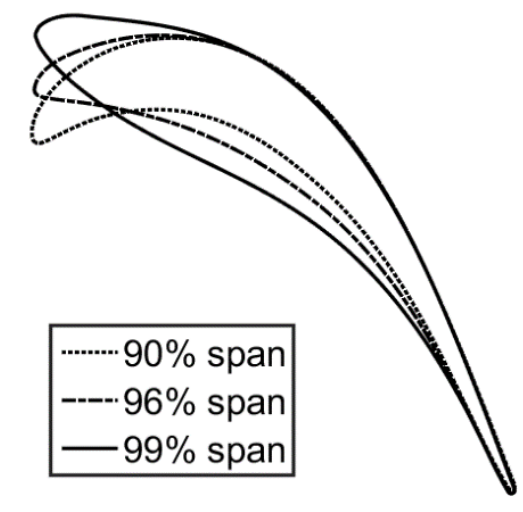

Fig. 14: New Stator 2 blade profiles at 90, 96 and $99 \%$ span.

Computational predictions: The calculated contours of Qcriteria within the New Stator 2 passage are shown in Fig. 15, for the non-dimensional deterioration value of $1.75 \%$. Only contours of Q-criteria with positive streamwise vorticity are shown. It is immediately apparent from examining the contours of Q-criteria and comparing with Fig. 11 that the separation vortex has been eliminated in the New Stator 2: the large vortex which stretched across the passage from the pressure side leading edge to the suction side trailing edge in Fig. 11 is no longer present. The blue streamlines were seeded on the surface of the middle stator of the three stators shown, and they do not separate from the surface.

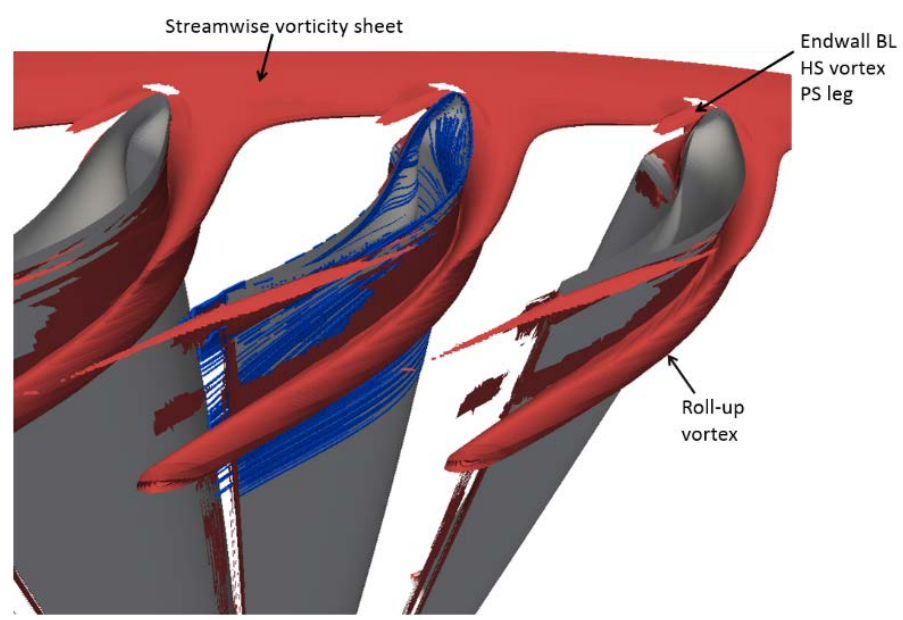

Fig. 15: Calculated contours of Q-criteria $\left(10 \times 10^{6}\right.$ to $20 \times$ $10^{6}$ ) with positive streamwise vorticity within the New Stator 2 passage for the non-dimensional deterioration value of $1.75 \%$. The blue streamlines were seeded on the blade surface of the middle stator. 


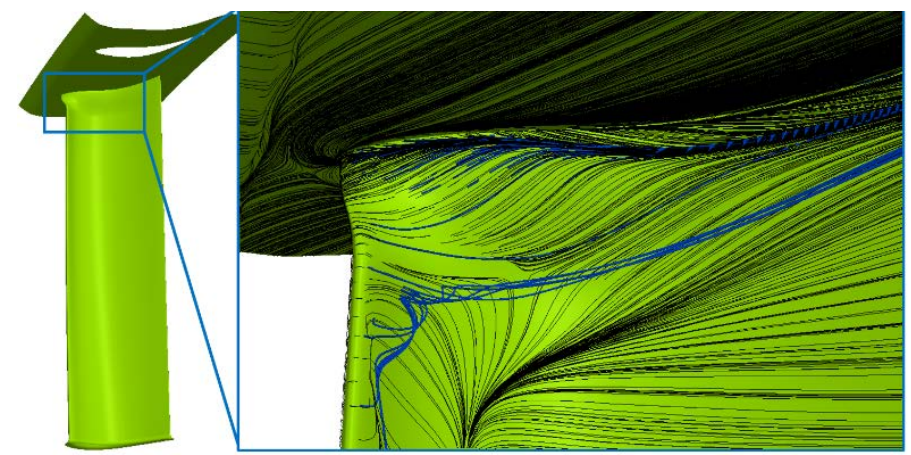

Fig. 16: A close-up of the pressure side leading edge of the New Stator 2 for the non-dimensional deterioration value of $1.75 \%$. The calculated surface skin friction lines are shown in black. The blue streamtubes were seeded on the blade surface.

A close-up of the pressure side leading edge of the New Stator 2 is shown in Fig. 16, for the non-dimensional deterioration value of $1.75 \%$. The surface skin friction lines are shown in black. The pattern of critical points seen in Fig. 13 is not present. The blue streamlines were seeded on the surface of the stator. The flow remains attached across the top 7\% of the span, but there is a small separation at approximately $90 \%$ span. Examining the skin friction lines of the casing endwall, it can be seen that the casing endwall boundary layer does not separate.

The calculated blade surface pressure coefficient for the Datum and New Stators at 96\% span for a non-dimensional deterioration of $1.75 \%$ is shown in Fig. 17. The large region of negative loading in the first fifteen percent axial chord is clearly visible for the Datum Stator 2.

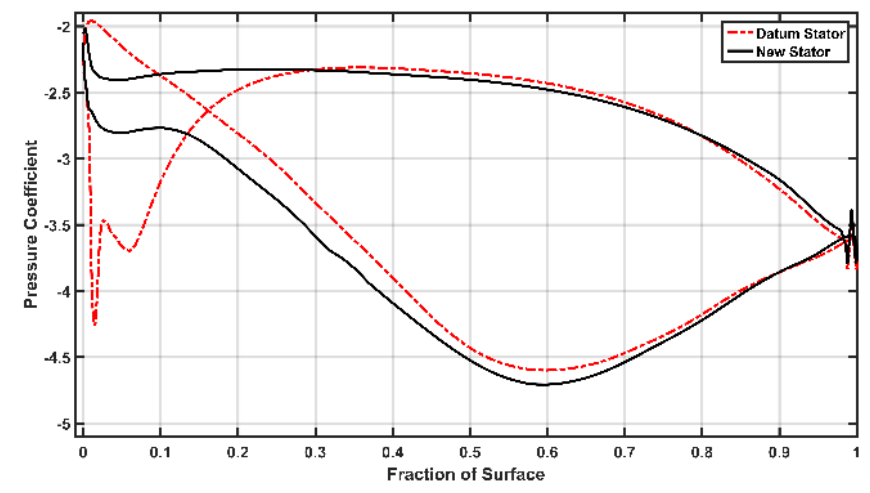

Fig. 17: Comparison of the calculated pressure coefficient for the Datum and New Stator 2 at $96 \%$ span for the nondimensional deterioration of $\mathbf{1 . 7 5 \%}$.

Evolution of the inlet streamwise vorticity: The calculated behaviour of the streamwise vorticity sheet within the Datum and New Stator 2 blade passages is now examined for the nondimensional deterioration value of $1.75 \%$. Sections covering 80 $100 \%$ span through the passages at 3\%, 7\%, 44\% and $84 \%$ axial chord are shown in Fig. 18. The development of the streamwise vorticity sheet is shown by the white stream-tracers which were seeded along a line at $100 \%$ span at the computational domain inlet (MP4). The contours of the non-dimensional static pressure identify the formation of the separation vortex on the pressure surface of the Datum Stator 2 at $7 \%$ axial chord. The contours also indicate the position of the separation vortex a $44 \%$ and $84 \%$ axial chord.
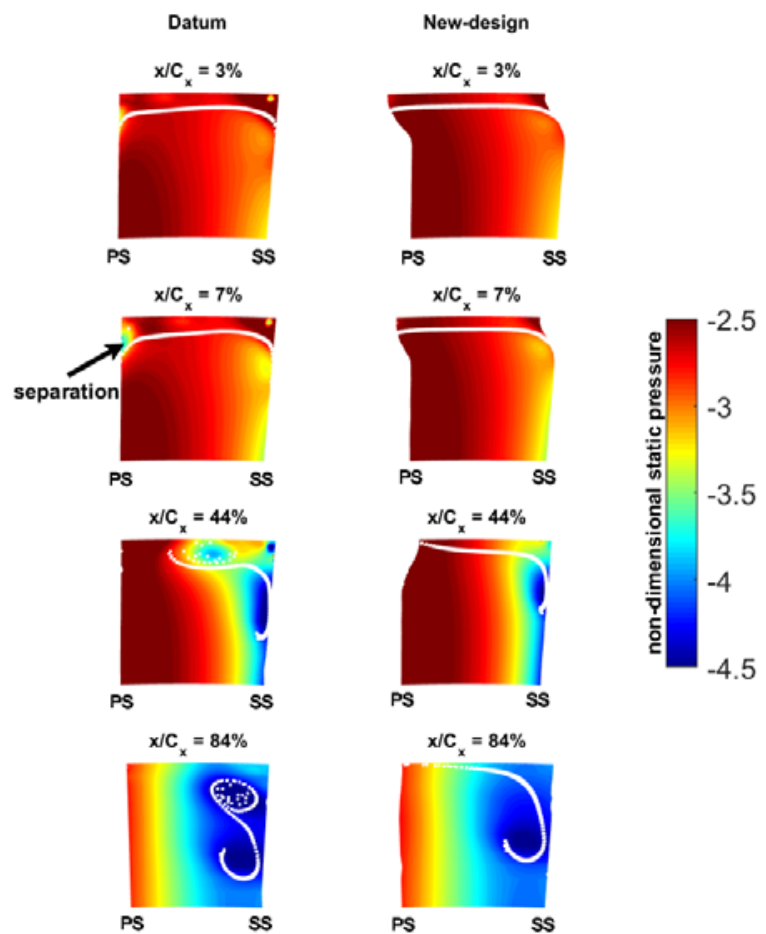

Fig. 18: Calculated evolution of the streamwise vorticity sheet through the Datum Stator 2 (left) and the New Stator 2 (right) for the non-dimensional deterioration of $1.75 \%$. The background contours are non-dimensional static pressure.

For both Stator designs the general motion of the streamwise vorticity sheet is towards and then down the suction surface, as indicated by the white stream-tracers. This is caused by the wall image vortex effect. For the Datum Stator 2, the pressure surface separation releases vortex filaments from the boundary layer which have the same rotation as the streamwise vorticity sheet. This enhances the rolling up of the streamwise vorticity sheet and causes part of it to wrap around the separation vortex causing it to strengthen. Therefore, two essentially distinct vortices are formed which would be expected to have a combined circulation greater than that of the streamwise vorticity sheet associated with the shroud-leakage. For the New Stator 2, which does not have the pressure surface separation, the streamwise vorticity sheet rolls up into a single vortex which would be expected to have similar circulation to that associated with the shroud-leakage.

\section{NEW STATOR 2 WITH ROTOR 1 DETERIORATION}

The New Stator 2 was manufactured and tested. In order to reduce time and cost overheads, only 16 New Stator 2 blades 
were installed (approximately 20\% of the annulus). The $16 \mathrm{New}$ Stators were equally spaced either side of the three stator pitches where the area traverses are performed. The periodicity across these three stator pitches was experimentally verified.

For the four values of the non-dimensional deterioration, the contours of the non-dimensional streamwise vorticity at the exit of the New Stator 2 (MP5) are shown in Fig. 19. Comparing these with those for the Datum Stator 2, Fig. 9, it can be seen that the New Stator 2 has successfully eliminated the separation vortex for all values of deterioration.

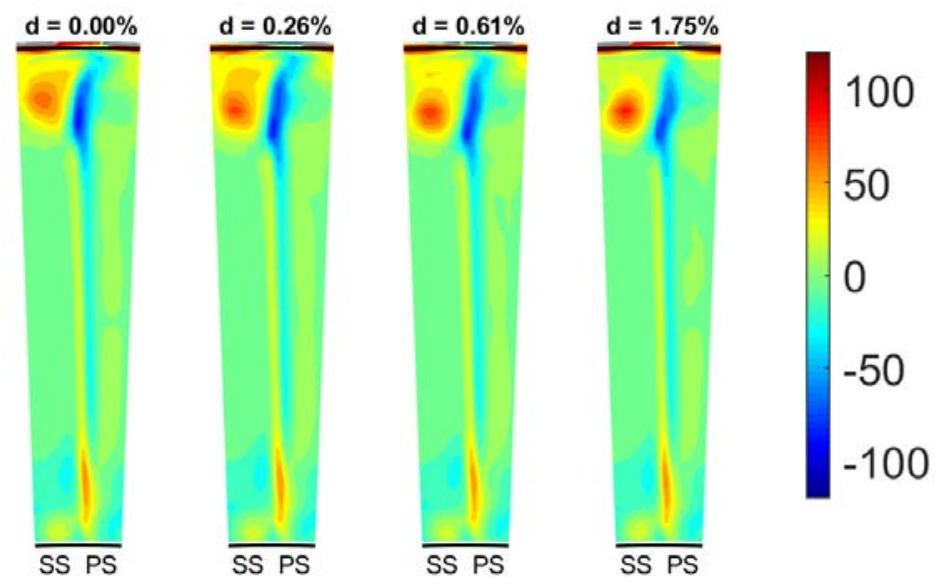

Fig. 19: Measured contours of non-dimensional streamwise vorticity at the exit of the New Stator 2 (MP5), for values of the non-dimensional deterioration.

Based on the computational studies and vortex kinematics, it has been hypothesised that the roll-up vortex is related to the strength of the sheet of streamwise vorticity associated with the Rotor 1 over-shroud leakage flow. A simple estimate of the nondimensional circulation of the streamwise vorticity associated with the shroud leakage flow is:

$$
\Gamma_{\text {streamwise }}=\frac{2 \pi}{N_{\text {stator }}} \frac{r_{\text {tip }}}{r_{\text {mean }}} \psi_{\text {stage } 1}
$$

where at rotor exit $\left(v_{\theta, \text { leak }}-v_{\theta \text {,main }}\right) \approx \Delta v_{\theta, \text { rotor }}=U \psi_{\text {stage } 1}$.

The non-dimensional circulation from the area traverses at the exit of the Datum and New Stator 2 passages has been determined using a contour around the positive vortices corresponding to a non-dimensional streamwise vorticity of 20 . For the Datum Stator 2, it was not possible to distinguish between the separation and roll-up vortices for the smaller deteriorations, so only the total has been obtained.

The total positive non-dimensional circulations for the Datum and New Stator 2 designs are compared in Fig. 20. For the New Stator 2 the circulation is virtually independent of the deterioration. This is consistent with the hypothesis that the rollup vortex is primarily created from the streamwise vorticity associated with the over-shroud leakage flow. The value of the estimated circulation, Eqn. 5, also agrees, to within 25\%, with that measured for the New Stator 2. For the Datum Stator 2 there is a dependence on the deterioration, especially for small deteriorations, and it is larger than that for the New Stator 2. This is consistent with the hypothesis that additional streamwise vorticity, associated with the separated pressure surface boundary layer, is introduced by the separation vortex which is larger at higher deteriorations (increased leakage flow and higher stagnation pressure)

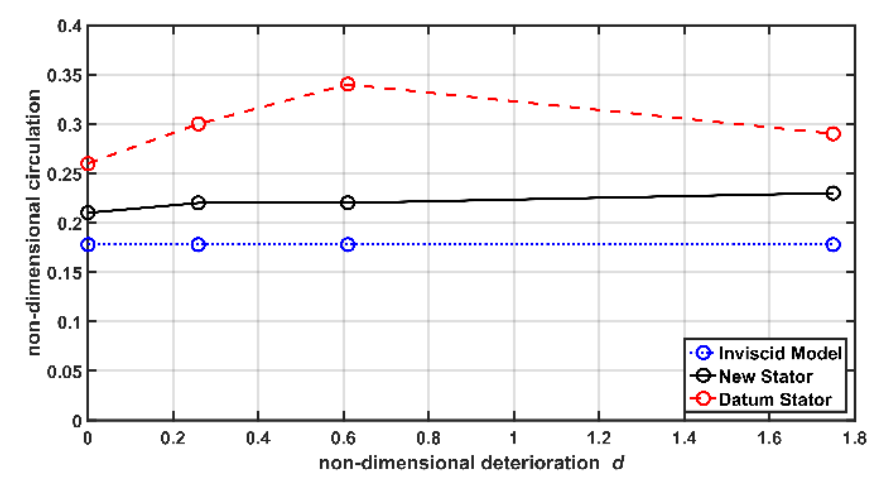

Fig. 20: Comparison of the measured circulation associated with the combined roll-up and separation vortices for the Datum Stator 2 with the roll-up vortex for the New Stator 2 (integrated around the 20 value streamwise vorticity contour). Also shown is the inviscid model circulation associated with the shroud leakage streamwise vorticity.
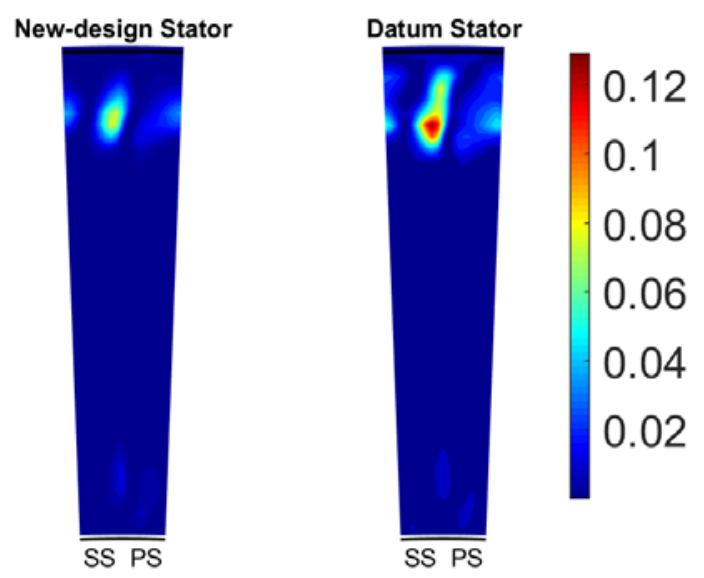

Fig. 21: Measured contours of the non-dimensional exit secondary kinetic energy for both New and Datum Stator 2 (MP5), for non-dimensional deterioration of $1.75 \%$.

Comparing Fig. 9 and Fig. 19 it is clear that the secondary flow field at the exit of the New Stator 2 is weaker than that for the Datum Stator 2, particularly for the non-dimensional deterioration value of $1.75 \%$. The contours of the nondimensional secondary kinetic energy at the exit (MP5) of the Datum and New Stator 2 for the non-dimensional deterioration value of $1.75 \%$ are shown in Fig. 21. In order to calculate the secondary kinetic energy the circumferentially averaged velocity components at each spanwise location were chosen to be the primary velocity components. The area of high secondary kinetic energy is smaller for the New Stator 2 and the maximum value is lower. Hence, the Rotor 2 will see a more uniform inlet flow with the New Stator 2. 
Performance of New Stator 2: For both Stator 2 designs the entropy loss coefficient for the four values of nondimensional deterioration for the streamtubes $0-100 \%$ and 70 $100 \%$ mass flow are shown in Fig. 22. For the $70-100 \%$ and the $0-100 \%$ mass flow streamtube the entropy loss coefficient is less across the New Stator 2 than the Datum Stator 2, for all values of deterioration.

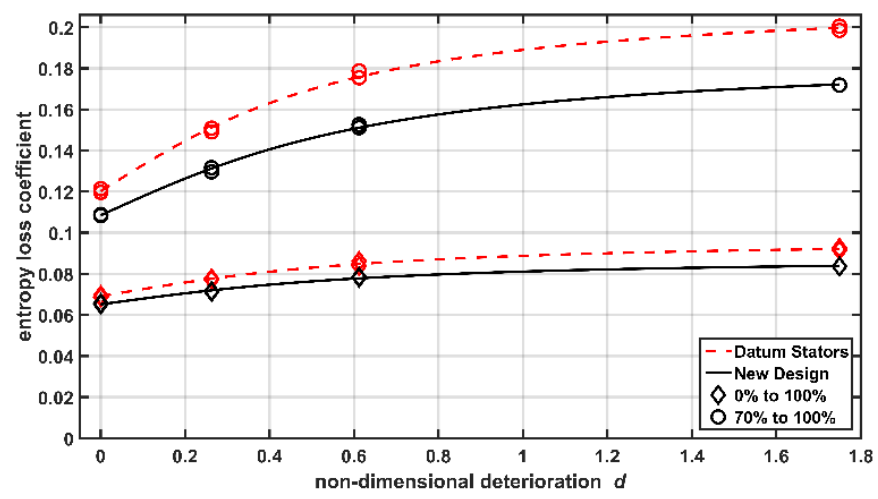

Fig. 22: Measured entropy loss coefficient for New Stator 2 (black) and Datum Stator 2 (red dashed) against nondimensional deterioration for the streamtubes $0-100 \%$ and $\mathbf{7 0 - 1 0 0 \%}$ mass flow (accounted using $\mathbf{3 0 - 4 0 \%}$ streamtube).

Because the Peregrine has high aspect ratio blading, the mainstream flow in the $0-70 \%$ mass flow streamtube is primarily unaffected by changes to the Rotor 1 deterioration. Hence, the entropy loss coefficient across the $70-100 \%$ mass flow streamtube is the most interesting. The increase in entropy loss coefficient across the $70-100 \%$ mass flow streamtube between the non-dimensional deterioration values of $0.00 \%$ and $1.75 \%$ has been reduced by $20 \%$ by the New Stator 2, relative to the Datum Stator 2 (also in Table 4).

Performance of Rotor 2 with New Stator 2: Because the Peregrine facility has two full stages, the effect of the Rotor 1 deterioration on the Rotor 2 performance can be investigated. However, only 16 of the New Design Stator 2 were installed (rainbow test) so only area traverse data are available. For the mainstream flow through Rotor 2, the change in the reduced relative stagnation pressure can be used to determine the entropy generation rate. This approach is not valid for the leakage flow over the Rotor 2 shroud. To minimise the associated error, the second stage knife-edge seal clearance was maintained at the design value. Therefore comparisons of the entropy generation rate across Rotor 2 are possible.

The entropy loss coefficient across Rotor 2 against the nondimensional deterioration for the streamtubes: $0-100 \%$ and 70 $100 \%$ mass flow is shown in Fig. 23 for both Stator 2 designs. For the $70-100 \%$ and the $0-100 \%$ mass flow streamtubes there is no difference in the Rotor 2 entropy loss coefficient between the two different Stator 2 designs for the non-dimensional deterioration value of $0.00 \%$ (new-condition). However, for larger values of deterioration Rotor 2 has a lower entropy loss coefficient when downstream of the New Stator 2 than when downstream of the Datum Stator 2. For the non-dimensional deterioration value of $1.75 \%$ (old-condition) the New Stator 2 has a $17 \%$ lower entropy loss coefficient across the $70-100 \%$ mass flow streamtube.

Furthermore, the entropy loss across Rotor 2 with the New Design Stator 2 can be seen to decrease with increasing deterioration in Fig. 23. As the deterioration increases the momentum of the leakage jet increases. For the New Design Stator 2 this momentum is not dissipated by the separation vortex. This reduces the momentum deficit in the tip portion at the Stator 2 exit (MP5) leading to Rotor 2 receiving a more uniform inlet profile (see [26] for more details).

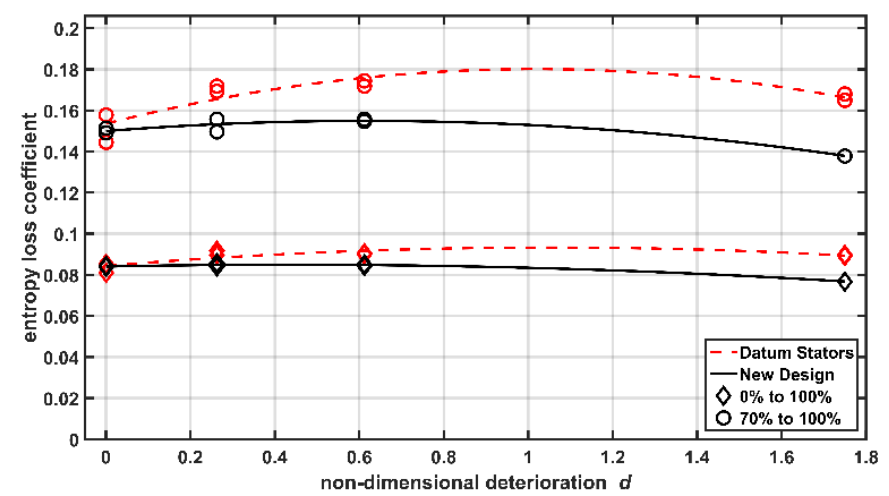

Fig. 23: Measured entropy loss coefficient across Rotor 2 when downstream of New Stator 2 (black) and Datum Stator 2 (red dashed) against the non-dimensional deterioration for the streamtubes $0-100 \%$ and $\mathbf{7 0 - 1 0 0 \%}$ mass flow (accounted using the $30-40 \%$ streamtube).

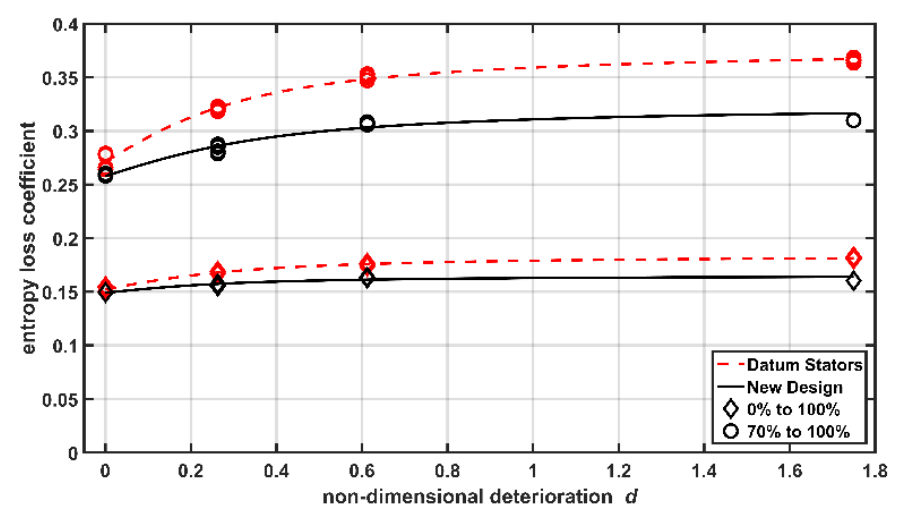

Fig.24: Measured entropy loss coefficient across Stage 2 for New Stator 2 (black) and Datum Stator 2 (red dashed) against non-dimensional deterioration for streamtubes $0-100 \%$ and $\mathbf{7 0 - 1 0 0 \%}$ mass flow (accounted using $\mathbf{3 0 - 4 0 \%}$ streamtube).

Performance of Stage 2 with New Stator 2: The entropy loss coefficient across stage 2 for both Stator 2 designs is shown in Fig. 24. The increase in the stage 2 entropy loss coefficient across the $70-100 \%$ mass flow streamtube between the nondimensional deterioration values of $0.00 \%$ and $1.75 \%$ has been reduced by $46 \%$ by the New Stator 2 compared with the Datum 
Stator 2. Furthermore, the aerodynamically integrated stage 2 work output was found to be unaffected by either the Rotor 1 leakage flow rate or the Stator 2 designs. Hence, the New Stator 2 improved the performance retention of the turbine.

\section{COMPARISON OF MEASURED AND CALCULATED}

The calculations captured the experimentally observed flow features, aided the interpretation of the relevant fluid mechanic mechanisms and were used to design the New Stator 2 which reduced the sensitivity to deterioration. Although not shown, the calculated trend in the entropy loss coefficient with deterioration was of similar form to those measured. A comparison between the measured and calculated increase in the stator 2 entropy loss coefficient for the $70-100 \%$ mass flow streamtube, i.e. tip region, are shown in Table 4. Although the calculations over predicted the performance benefit, the New Stator 2 was successful.

Only Stage 2 was calculated and the assumptions made were: steady-state, mixing-plane and fully turbulent boundary layers. Quantitatively, the calculated levels of vorticity are higher than those measured, see Figs. 9 and 10. (This may be due to the five-hole probe measuring some form of time-average of the unsteady flow field). These higher levels of streamwise vorticity may have resulted in more secondary kinetic energy in the calculations and hence the reason for the over prediction of the benefit.

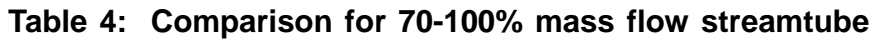
between the measured and calculated increase in the stator 2 entropy loss coefficient between the newcondition ( $d=0.00 \%)$ and old-condition ( $d=1.75 \%)$.

\begin{tabular}{lcc}
\hline & Measured $\Delta \zeta$ & Calculated $\Delta \zeta$ \\
\hline Datum Stator 2 & 0.080 & 0.060 \\
New Stator 2 & 0.064 & 0.028 \\
& $-20 \%$ & $-53 \%$ \\
\hline
\end{tabular}

\section{DISCUSSION}

In-service deterioration in multi-stage turbines introduces two important aspects associated with the increased clearance gap between the rotor shroud knife-edged seals and the casing. Firstly, the increase in the shroud-leakage mass flow rate; this has been extensively studied by previous researchers. Secondly, any variation in the angle at which the leakage flow approaches the downstream stator; this is the primary focus here.

Fluid mechanics: The measurements presented show that for deterioration from a new-condition to an old-condition the angle at which the shroud-leakage flow approaches the downstream stator changes by approximately $10^{\circ}$ but is $60^{\circ}$ to $80^{\circ}$ different from that of the mainstream flow. It is also relatively insensitive to the operating point (flow coefficient).

The engine representative chamfer at the exit of the rotor shroud cavity allows the over-shroud leakage flow to re-enter the mainstream with minimal mixing. The large difference between the tangential velocity of the over-shroud leakage flow and the mainstream flow means that there is a sheet of streamwise vorticity which enters the downstream stator row. Because of the presence of the stator blade surfaces and a Kelvin-Helmholtz style instability the sheet of streamwise vorticity rolls up to form a concentrated roll-up vortex which lies close to the suction surface in the endwall region. In the case studied here, the deterioration increases the difference between the mainstream and the over-shroud leakage tangential velocities by only approximately 20\%. Consequently, the strength of the roll-up vortex only increases moderately during deterioration. This is shown in Fig. 20 for the New Stator 2.

Depending on the design of the downstream stator, the large difference between the mainstream and the over-shroud leakage flow directions may cause a separation on the pressure surface close to the leading edge and endwall region due to the negativeincidence. For the Datum Stator 2 geometry investigated here, the negative loading, associated with the large negative incidence of $60^{\circ}$ to $80^{\circ}$, produced a separation vortex from the separated pressure-surface boundary layers. The strength of the separation vortex increases through two mechanisms. Firstly, the size of the leading edge separation increases with deterioration so more boundary layer vortex filaments can be released from the blade surface. Secondly, the increased positive vorticity enhances the rolling up process and more of the streamwise vorticity sheet is wrapped around the separation vortex. For the Datum Stator 2 studied here, for the new-condition the separation vortex could barely be distinguished from the roll-up vortex whilst for the old-condition it was clearly distinct.

The over-shroud leakage flow approaches the downstream stator at essentially a fixed angle which is independent of the operating point and deterioration. This observation means that in the endwall region the stator may not require a large amount of incidence tolerance. Therefore it may be possible to locally redesign the endwall region of that blade to reduce the large negative incidence. In the current investigation, a New Stator 2 was designed which both computationally and experimentally did not generate the separation vortex even for the old-condition and resulted in lower entropy loss coefficients.

Vorticity kinematics: For transverse vorticity entering a stator passage, the horseshoe vortex model for secondary flow predicts that there will be a single positive vortex at the exit of the blade row, the passage vortex, and a second vortex which is smaller and of opposite sign, the counter vortex. Although these are present in the stator 2 endwall region they are smaller than the two large positive vortices observed at the exit of the Datum Stator 2, see Fig. 9. The two large positive vortices are caused by different mechanisms and, in this paper, are referred to as the roll-up vortex and the separation vortex.

The roll-up vortex will always be present because it is fundamentally linked with the sheet of streamwise vorticity associated with the over-shroud leakage flow. The separation vortex may or may not be generated depending on the relationship between the stator blade design and the over-shroud leakage flow direction. 
Effect on average lifetime performance: For the newcondition both the Datum and the New Stator 2 designs give similar stage 2 efficiency. With the Datum Stator 2, the efficiency of the second stage reduces by about $2 \%$ due to the deterioration of the Rotor 1 shroud clearance to that corresponding to the oldcondition. However, with the New Stator 2, the efficiency of the second stage only reduces by about $1 \%$ between the newcondition and old-condition. The lifetime average performance of the second stage is approximately $0.5 \%$ higher. This would correspond to an annual saving of \$35,000 for an aero-engine with a bypass ratio of six.

\section{CONCLUSIONS}

Based on the aero-engine representative rotor shroud cavity geometry investigated here with either the Datum Stator 2 or New Stator 2 closely positioned downstream:

\section{Characteristics of the over-shroud leakage flow:}

- The rotor over-shroud leakage flow has a yaw angle which is more than $60^{\circ}$ higher than the mainstream flow angle.

- The leakage yaw angle is insensitive to flow coefficient.

- In-service deterioration from the new-condition to oldcondition reduces the leakage flow yaw angle by about $10^{\circ}$.

- The above are consistent with an extended version of Denton's over-shroud leakage model.

- Compared to the mainstream flow downstream of the rotor, the stagnation pressure of the leakage flow is higher and increases by approximately 2.4 working section dynamic heads $\left(\frac{1}{2} \rho v_{\text {axial }}^{2}\right)$ during deterioration.

Flow field ahead of the stator:

- For a cavity geometry which smoothly re-introduces the leakage flow back into the mainstream there is little mixing ahead of the downstream stator.

- The strong spanwise gradient in tangential velocity between the leakage and mainstream flows results in a sheet of streamwise vorticity entering the downstream stator.

Roll-up vortex:

- Within the stator passage the sheet of streamwise vorticity rolls up on the suction side of the stator passage and forms the roll-up vortex. (The mechanism is different from the horseshoe vortex model for secondary flow.)

- The roll-up vortex is a fundamental consequence of the rotor over-shroud leakage flow.

- The strength of the roll-up vortex is relatively insensitive to deterioration and it cannot be eliminated.

\section{Separation vortex:}

- The shroud-leakage approaches the Datum Stator 2 with more than $60^{\circ}$ of incidence and separates off the pressure side of the leading edge and some of the pressure side boundary layer vorticity is shed. These vorticity filaments form the basis of a separation vortex which also has some of the inlet streamwise vorticity sheet wrapped around it.

- The separation vortex is generated within the stator passage and increases in size with deterioration.

\section{Elimination of the separation vortex:}

- The New Stator 2 had a redesigned outer portion of the leading edge to reduce the incidence of the shroud leakage flow and the separation vortex was eliminated.

- It is unknown if it is possible to sufficiently thicken the stator leading edge, without changing the incidence, to avoid the separation vortex at large values of deterioration.

Potential benefits by eliminating the separation vortex:

- The estimated lifetime average performance retention due to the New Stator 2 design is $0.5 \%$ LPT efficiency.

\section{NOMENCLATURE}

$$
\begin{aligned}
& b=\text { non-dimensional blade speed, } U / \sqrt{c_{p} T_{0, \text { ref }}} \\
& c=\text { non-dimensional clearance, } \text { gap/span } \\
& c_{p}=\text { specific heat capacity at constant pressure } \\
& d=\Delta \text { gap } / \text { span } \\
& g=c_{p} / R \\
& \text { gap = clearance between rotor knife-edge seal and casing } \\
& \Delta h_{0}=\text { stagnation enthalpy drop } \\
& p, p_{0}=\text { static, stagnation pressure } \\
& r=\text { radius } \\
& R=\text { gas constant } \\
& s=\text { specific entropy } \\
& T, T_{0}=\text { static, stagnation temperature } \\
& U \text { = blade speed } \\
& v_{x}, v_{\theta}=\text { axial, tangential velocity } \\
& \alpha=\text { yaw angle } \\
& \phi \text { = flow coefficient, } v_{x, \text { ref }} / U \\
& \psi=\text { stage loading, } \Delta h_{0} / U^{2} \\
& \gamma=\text { pitch angle } \\
& \rho=\text { density } \\
& \zeta=\text { entropy loss coefficient, } T_{0, r e} \Delta s / U^{2}
\end{aligned}
$$

\section{ACKNOWLEDGMENTS}

The Authors would like to thank Innovate UK and RollsRoyce for funding this work. The discussions with Dr C. Clark, Mr F. Goenaga, Dr K. Evans and Dr K. Millidonis are gratefully acknowledged as are the skills of Mr D. Basham and Mr B. Coles in the manufacturing of the necessary components.

\section{REFERENCES}

[1] Chapman, J. W., Guo, T.-H., Kratz, J. L., and Litt, J. S., 2016. Integrated turbine tip clearance and gas turbine 
engine simulation. In 52nd AIAA/SAE/ASEE Joint Propulsion Conference, page 5047.

[2] Lattime, S. and Steinetz, B., 2002, Turbine engine clearance control systems: current practices and future directions. In 38th AIAA/ASME/SAE/ASEE Joint Propulsion Conference \& Exhibit, page 3790.

[3] Cumpsty, N. A., 2004, Compressor Aerodynamics, Krieger, Malabar, FL.

[4] Denton, J.D., 1993, IGTI Scholar Lecture: Loss Mechanisms in Turbomachines, J. Turbomach. 115(4): 621-656 (36 pages).

[5] Peters, P., Breisig, V., Giboni, A., Lerner, C., and Pfost, H., 2000, The influence of the clearance of shrouded rotor blades on the development of the flowfield and losses in the subsequent stator. In ASME Turbo Expo 2000: Power for Land, Sea, and Air, pages V001T03A057-V001T03A057. American Society of Mechanical Engineers.

[6] Wallis, A., Denton, J., and Demargne, A., 2001, The control of shroud leakage flows to reduce aerodynamic losses in a low aspect ratio, shrouded axial flow turbine. J. Turbomach, 123(2):334-341.

[7] Rosic, B., Denton, J. D., and Curtis, E. M., 2008, The influence of shroud and cavity geometry on turbine performance: an experimental and computational studypart I: shroud geometry. J. Turbomach, 130(4):041001.

[8] Rosic, B., Denton, J. D., Curtis, E. M., and Peterson, A. T., 2008, The influence of shroud and cavity geometry on turbine performance: An experimental and computational study-part II: Exit cavity geometry. J. Turbomach, 130(4):041002.

[9] Pfau, A., Kalfas, A., and Abhari, R., 2007, Making use of labyrinth interaction flow, J. Turbomach, 129(1):164174

[10] Curtis, E. M., Denton, J. D., Longley, J. P., and Rosic, B., 2009, Controlling tip leakage flow over a shrouded turbine rotor using an air-curtain. ASME Turbo Expo 2009: Power for Land, Sea, and Air, pages 885-894.

[11] Rosic, B. and Denton, J. D., 2008, Control of shroud leakage loss by reducing circumferential mixing. $J$. Turbomach, 130(2):021010.

[12] Gao, J., Zheng, Q., Yue, G., and Sun, L., 2012, Control of shroud leakage flows to reduce mixing losses in a shrouded axial turbine. Proceedings of the Institution of Mechanical Engineers, Part C: Journal of Mechanical Engineering Science, 226(5):1263-1277.

[13] Evans, K. R. and Longley, J. P., 2017, Clocking in LowPressure Turbines. J. Turbomach, 139(10):101003 (15 pages).

[14] Longley, J. P., 2019, Modelling the unsteady dynamics of a turbine research facility. In ASME Turbo Expo 2019: Turbomachinery Technical Conference and Exposition. Phoenix, AR

[15] Taylor, D., and Longley, J. P., 2019, Effects of Stator Platform Geometry Features on Blade Row Performance. J. Global Power and Propulsion Society, 3, (20 pages).
[16] Evans, K. R. and Longley, J. P., 2017, Accounting for Uncontrolled Variations in Low-Speed Turbine Experiments, J. Turbomach, 139(10): 101005 (12 pages)

[17] Lapworth, L., 2004, Hydra-cfd: a framework for collaborative CFD development. In International Conference on Scientific and Engineering Computation (IC-SEC), Singapore, June, volume 30.

[18] Milidonis, D. K., 2016, Steady-state two-stage calculations of the Peregrine Rig.

[19] Yoon, S., Curtis, E., Denton, J., and Longley, J., 2014, The effect of clearance on shrouded and unshrouded turbines at two levels of reaction. J. Turbomach, 136(2):021013.

[20] Holmen, V., 2012, Methods for vortex identification. Master's Theses in Mathematical Sciences.

[21] Inoue, M., Kuroumaru, M., Tanino, T., and Furukawa, M., 2000, Propagation of multiple short-length-scale stall cells in an axial compressor rotor. J. Turbomach, 122(1):45-54.

[22] Pullan, G., Young, A., Day, I., Greitzer, E., and Spakovszky, Z., 2015, Origins and structure of spike-type rotating stall. J. Turbomach, 137(5):051007.

[23] Giboni, A., Wolter, K., Menter, J.R. and Pfost, H., 2004, Experimental and numerical investigation into the unsteady interaction of labyrinth seal leakage flow and main flow in a 1.5-stage axial turbine. In ASME Turbo Expo 2004: Power for Land, Sea, and Air (pp. 983-992). American Society of Mechanical Engineers Digital Collection.

[24] Gbadebo, S. A., Cumpsty, N. A., and Hynes, T. P., 2005, Three-dimensional separations in axial compressors. J. Turbomach, 127(2):331-339.

[25] Batchelor, G. K. (2000). Flow of Effectively Inviscid Fluid with Vorticity, page 507-593. Cambridge Mathematical Library. Cambridge University Press.

[26] Jameson, H. K., 2019, The effects of shrouded rotor tip leakage flow on the downstream stator aerodynamics in turbines. Cambridge University. Ph.D. thesis, Cambridge University, Cambridge, UK.

\section{APPENDIX: INVSCID MODEL FOR FLOW ANGLE OF LEAKAGE JET}

Denton [4] presented a simple model to estimate the shroud leakage mass flow rate across a single knife-edge seal. Three assumptions were made: isentropic flow up to the seal throat, uniform static pressure downstream of the seal and that the tangential velocity of the leakage flow above the shroud would not be greatly changed. Denton outlined that downstream of the seal throat the leakage jet would mix within the shroud cavity and that further mixing would occur when the leakage flow was re-introduced into the mainstream (the two flows have different meridional and tangential velocity components).

The same three assumptions will be made here but, because of the chamfered exit geometry of the shroud cavity (see Fig. 2), it will be assumed that there is no significant mixing until the leakage flow has re-entered the mainstream. A schematic of the 
assumed flow through the leakage path is shown in Fig. 25. By assuming that there is little mixing within the shroud cavity it is possible not only to estimate the leakage velocity at the seal throat but also where the flow re-enters the mainstream.

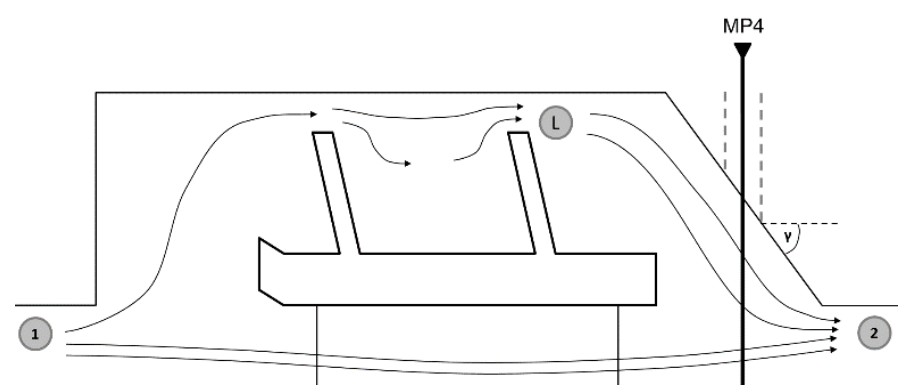

Fig.25: A schematic the flow through the leakage path, assuming inviscid flow (no mixing in the leakage path). Three points are labelled: point 1 is upstream of Rotor 1, point $L$ is in the leakage jet immediately downstream of the final knife-edge seal and point 2 is downstream of Rotor 1.

Working in the relative frame (but ignoring the effects of any radius change) the relative tangential velocity of the leakage flow above the shroud is given by:

$$
v_{\theta 1, \text { leak }}^{\text {rel }}=v_{\theta 1, \text { main }}-U=\left(\phi \tan \alpha_{1}-1\right) U
$$

Isentropic leakage flow from upstream, 1, to downstream, 2, gives:

$$
p_{2, \text { leak }}=p_{01}^{\text {rel }}-\frac{1}{2} \rho\left(v_{m 2, \text { leak }}^{2}+\left(v_{\theta 2, \text { leak }}^{\text {rel }}\right)^{2}\right)
$$

For the mainstream flow, which is also taken to be isentropic:

$$
p_{2, \text { main }}=p_{01}^{\text {rel }}-\frac{1}{2} \rho v_{x 2 \text {,main }}^{2}\left(1+\tan ^{2} \alpha_{2}^{\text {rel }}\right)
$$

The assumption of uniform static pressure across the shroud exit cavity and mainstream downstream of the rotor:

$$
v_{m 2, \text { leak }}^{2}=v_{x 2 \text {,main }}^{2}\left(1+\tan ^{2} \alpha_{2}^{\text {rel }}\right)-\left(v_{\theta 1, \text { leak }}^{\text {rel }}\right)^{2}
$$

where it has been assumed that $v_{\theta 2 \text {,leak }}^{\text {rel }}=v_{\theta 1, \text { leak }}^{\text {rel }}$. Rearranging the above gives:

$$
v_{m 2, \text { leak }}^{2}=\phi^{2} U^{2} \frac{1}{\cos ^{2} \alpha_{2}^{\text {rel }}}-\left(\phi \tan \alpha_{1}-1\right)^{2} U^{2}
$$

The above can be re-written as:

$$
v_{\text {m2,leak }}=\phi U \sqrt{\frac{1}{\cos ^{2} \alpha_{2}^{\text {rel }}}-\left(\tan \alpha_{1}-\frac{1}{\phi}\right)^{2}}
$$

The absolute flow angle of the over-shroud leakage flow as it reenters the mainstream is given by:

$$
\tan \alpha_{2, \text { leak }}=\frac{v_{\theta 2, \text { leak }}}{v_{x 2 \text {,leak }}}=\frac{v_{\theta 1, \text { leak }}}{v_{m 2, \text { leak }} \cos \gamma}
$$

where $\gamma$ is the inclination of the chamfer at the cavity exit (as indicated on Fig. 25). The above two equations can be combined to give:

$$
\tan \alpha_{2, \text { leak }}=\frac{\tan \alpha_{1}}{\cos \gamma \sqrt{\frac{1}{\cos ^{2} \alpha_{2}^{\text {rel }}}-\left(\tan \alpha_{1}-\frac{1}{\phi}\right)^{2}}}
$$

